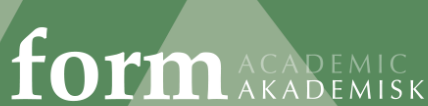

Vol I3, No 4 (2020) https://doi.org/10.7577/formakademisk.3379

Athina Stamatopoulou

MSc Architect, PhD candidate

School of Architecture, Department of Architectural Language, Communication and Design, National Technical University of Athens athinastamatopoulou@gmail.com

\title{
Design for Relations
}

Developing a methodology of mapping and designing in the city as an open, complex system

\begin{abstract}
The city, composed of heterogeneous relations, is an open complex system, beyond its physicality. Questioning analysis and design, a work-in-progress methodology of a generative mapping negotiating different kinds of design logics is presented. The methodology is composed of (1) data gathering; (2) investigating relations among data; and (3) testing with two cases elicited from Athens Center public space. The focus is on how such a relational-thinking methodology cultivates design logics through designing by analyzing; designing by defining frame(s); designing the program; designing as an interventional strategy in/through interdependencies; designing by intervening with territorial forces; and employing intra-parametric strategies. By enforcing emergence and management of complexities in diverse fields of potential application, the methodology negotiates the limits and the ways of design.
\end{abstract}

Keywords:

Systemic urban design, relational design, relational territorialities, relationally generated complexity; design methodology; open method; diagrammatic mapping

\section{INTRODUCTION}

In this paper a work is presented, which is part of a dissertation in progress, on the development of a methodology for research and analysis, description, and design in the city from a relational point of view. Relationality prioritizes the approaches to things through their relations ${ }^{1}$. The conception of relations in the paper is affiliated with the condition of assemblages/agencements, drawing from the Deleuze and Guattari (1987/1980) and the DeLanda (2016) philosophies. Emphasis is given to the composition of relations by heterogeneous elements, further accompanied by diversity, multiplicity, and change. In this paper, relational and system thinking converge under the common denominator of complexity (i.e. Cilliers, 1998 and 2001). Hence, the city's complexity is articulated with its multiplicity and relationality, by being conceived of as an assemblage composed of dynamic, constantly changing, and multiple relations among heterogeneous things. These relations are defined by diverse parameters, both material and immaterial (i.e. socio-economic conditions, information, events). For instance, a 
bench alongside a street can be approached through its material form and structure. By thinking about its functions and placement in the city, the approach disengages from physicality. It becomes a field of forces, rather than a neutral object. Adding data on how it interacts with its milieu, inserts socially and culturally oriented parameters. Its symbolical role(s) and attributed meanings to it reveal more aspects, contributing to the complex relationality of the bench.

Be it a bench or a city, the complexity of things triggers questions (1a in Figure 1) as to how they can be understood, explored, and communicated. Such questions expand to analysis and design in the city: The research question is, "How might analysis and design-in their generative interlacingintegrate conditions triggered by complexity, relationality, and constant change?" By emphasizing how, the question becomes a methodology challenge, when approaching the city, both for analysis and design, or largely for theory and practice. In this context, design by being linked to analysis, theory, and research amplifies the question toward ways of what is argued here as analyzing and designing (1b in Figure 1). In parallel, looking at the city beyond its physicality, namely as a physical and cultural object carrying ideologies, values, and meanings, constitutes a question of description, for its conception and multimodal communication. For this reason, the semiotic complexity of the city is prioritized.

The research objective is the development of an open methodology (1c in Figure 1) for analyzing and designing in the city with regard to its semiotic relationality-complexity. The development of the methodology is in progress. It is inspired by the research by design (1d in Figure 1) that enhances synergies between research and design (Sevaldson, 2010) as well as reflection and action (Schön, 1983). Specifically, the methodology evolves through the dialogue of a theoretical and a practice-oriented part, which contains testing applications and experiments. In the project's wider field, the paper is focused on a phase of the methodology's development. Considering the key role of the experiments, consideration is given to the research by examining how the combinatory view of two different tests (in Athens public space; 1e in Figure 1) gives feedback to the methodology's theoretical part, and mainly to its aims toward design processes (1f in Figure 1). Research by design as well as the dynamic complexity of a city suggest the methodology's openness: openness for improvisation; openness for the method's flexibility to change; and for adaptability to diverse contexts. The objective of this research is clarified through the analysis of its aims and intended contributions.



FIGURE 1. Visual abstract of the paper (visualization: Stamatopoulou, 2020). 
First, by applying this methodology when analyzing and designing in the city, one can go beyond its physical-material level, without detaching from it. The semiotic and the physical networks are interlinked. For example, two parks in a city are located at a distance without a direct physical relationship. At the same time, three individuals agree that they perceive them as "attractive parks with impressive flora" in a way that enables thinking of these locations ${ }^{2}$ as interrelated with regard to the attributed qualities. The methodology is expected to take into account both kinds of information. Thus, this objective expands to the aim of interweaving diverse materialities (i.e. parks' flora) and nonmaterialities (i.e. their impressiveness and attractiveness). This contributes to the integration of qualitative relations in the analysis and design processes, as well as in the merging of quantities and qualities. Quantities may refer to the distance and size of the aforementioned parks, while qualities include all semiotic data. The methodology gives access to any location through the system of diverse properties: In this system, the distance and size of the park affect the attractiveness of their flora, and vice-versa. Secondly, every action in this process is contemplated to be creative, taking nothing for granted, debating established certainties, and breaking preconceptions. By this, a critical dimension is embedded in the methodology.

Both intensions envision the capability of analyzing the city with regard to the parameters of its relationality-complexity. In broad applications of the methodology, these parameters of relations are context- and case-dependent, in order to keep the methodology open. The existence of (any) parameters is sufficient for the intended generative analysis. Parameters allow for de-composing and recomposing the city, beyond its established form. Instead of restricting the thinking to that of its physical form, with the proposed methodology, different assemblages of it are enforced. Operations in Figure 2 illustrate how the city's physical aspects (map 1a) can be (re)organized in multiple ways with regard to semiotic relations. Re-composition options for a city's re-organization originate from the blue networks of map 1b. The re-composition of the city is shown in maps 2 and 3 . Distances among locations on maps $2 a$ and $3 a$ keep their physical dimensions. Maps $2 b$ and $3 b$ are an experiment with physically distorted re-compositions, resulting from semiotic attractions (based on blue arrows of maps $2 \mathrm{a}$ and $2 \mathrm{~b}$ ) among shifted relations of locations. In these re-organizations, looking beyond the physical, by de-composing it, its significance is not doubted; in fact, its value is augmented by the triggering of multiple variations of it.

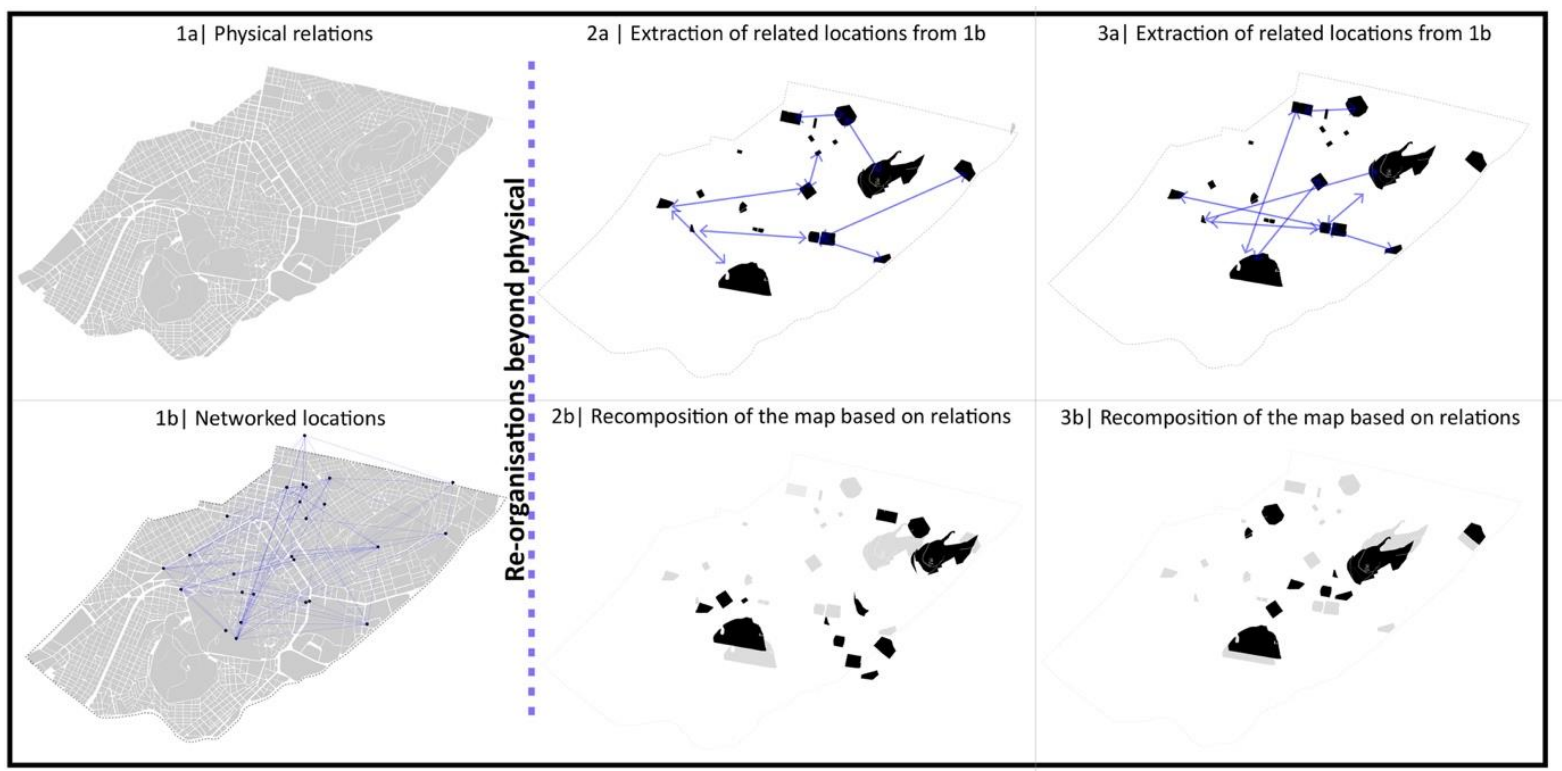

FIGURE 2. Co-consideration of semiotic-physical relations: de-composition of the physical and re-composition into new (shifted) relations among locations $(2 \mathrm{~b}, 3 \mathrm{~b})$. Gray/black in maps $2 \mathrm{~b}$ and $3 \mathrm{~b}$ : physical relations/shifted ones, respectively (visualization: Stamatopoulou, 2020). 
In this context of intentions (Figure 3, left part), the methodology is aimed at creating a tool for gathering and combining the methodology's intended capacities, in order to feed relations-oriented design processes. Design is conceived of as creation of the design processes and strategies, similar to systemic design (Jones, 2014) and medium design (Easterling, 2017).

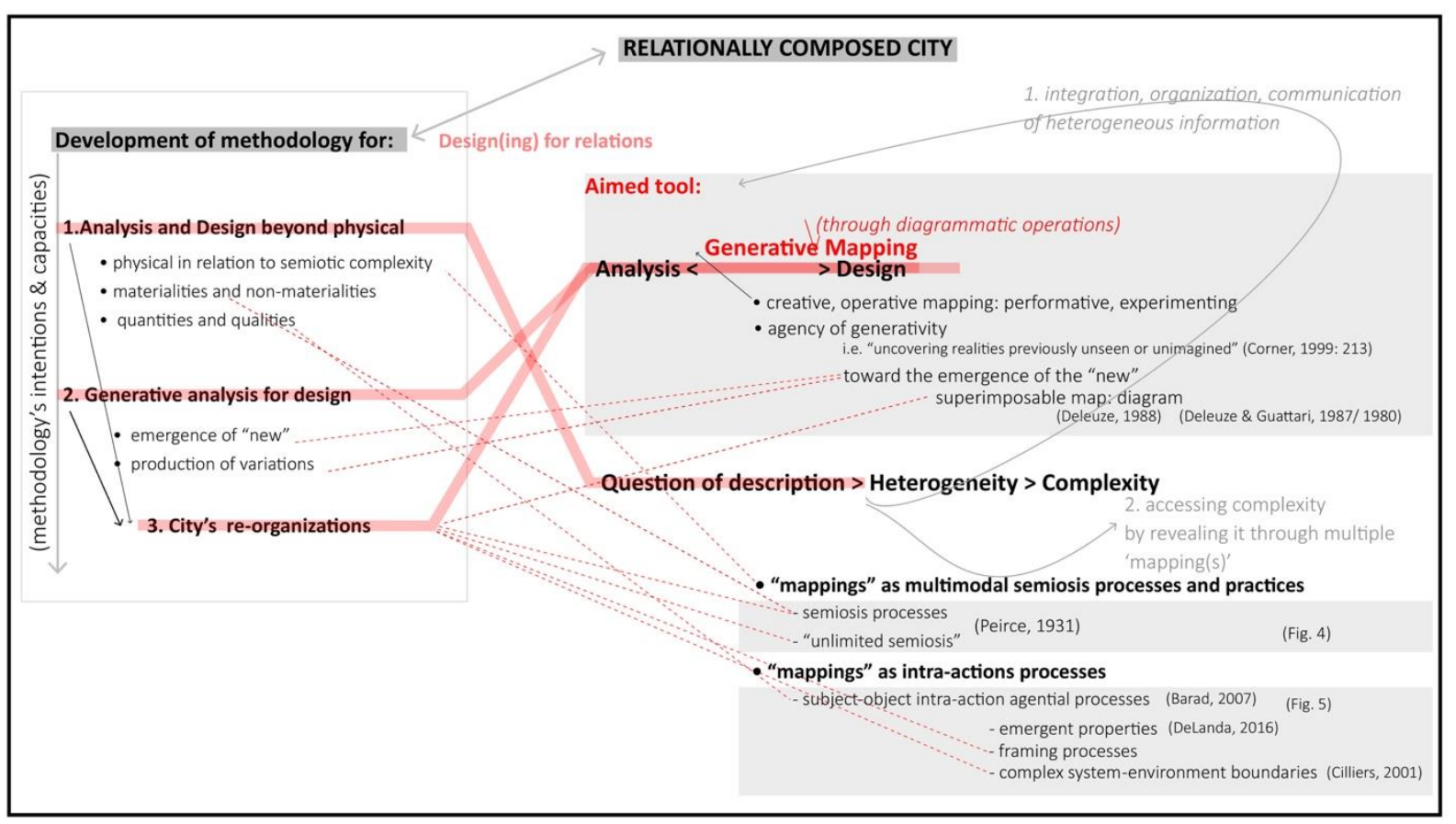

FIGURE 3. Scheme of relations among the methodology's intentions-capacities (left side), and how these are served by the tool and its features (visualization: Stamatopoulou, 2020).

With the research hypothesis, it was assumed that a mapping tool may be used to negotiate the gathering and synergies of the intended capacities, and through these, organize relations among analysis and design: The aim for a generative -through diagrammatic operations-mapping was set as central. The intended features of this diagrammatic mapping are further specified, with regard to how they serve the methodology's capacities, within the research frame.

\section{Mapping as complex, generative, diagrammatic operation}

Mapping can mediate analysis and design synergies, if seen as an operative and creative process (Paez, 2019; Corner, 1999). Such mappings are generative by being performative ${ }^{3}$ and by experimenting (Paez, 2019, p. 24). For James Corner, creative mappings are driven by agency: Agency lies on their generative capacities, such as "uncovering realities previously unseen or unimagined" (Corner, 1999, p. 213). These capacities are associated with the emergence of the new. ${ }^{4}$ New might emerge through the interrelationships of what already exists, through the possible, and the unveiling of potentials. It can be achieved through the "superimposable map" (Deleuze, 1988): Superimposed maps operate new connections and indicate new potentialities (Zdebik, 2012). Based on these, the diagram, conceived through Deleuze's (1988) account, is a machine that can "construct new types of realities" (Deleuze and Guattari, 1987, p. 142). These can be produced not only by the coexistence of diverse realities, but through their interactions. In this context, the aimed mapping is such a machine capable of diagrammatizing, in order to experiment with the generativity of relations as well as with their organization. The tool's generative capacities contribute to the revealing of complexity, its understanding, and its exploration. At the same time, by embedding diagrammatic operations, the tool enables the management of complexity through abstractions, which are still generative (Massumi, 
2011, p. 14). ${ }^{5}$ This generative diagrammatic mapping tool is further framed through constraint features, defined by the research question regarding description.

\section{Mappings as multimodal semiosis processes and practices}

The question of description triggers how the aimed mapping may be used to integrate heterogeneous data (i.e. with regard to content and means of representation), as well as organize and communicate their relations. At the same time, heterogeneity/multiplicity is a milieu for accessing complexity (i.e. bench example). Therefore, the tool is informed by multiple (sub)mappings (reference following as mappings or mapping). Each mapping is conceived of as a description, expressed through any media, i.e. written text, song, movie, image, or model. By examining them both as processes and as practices, attention is given to their context and the ways in which they were created, in addition to their visual result.

In parallel, each mapping is a semiosis process, under the terms of C. S. Peirce (1931) and his triadic relational model of semiosis (Figure 4). A sign/semiosis is composed of three interrelated terms. The first is a representamen (different from "representation") of a second term, that is the referent/ object to which this is referred in a way that allows for the defining of a third term, the interpretant (Peirce, 1931). For instance, a public square (object/referent) signifies "home" (interpretant), according to a subject (of a semiosis process) who connects it (the public square) with its "childhood memories" (representamen). Through repetition of the process, the public square is open to further semiosis, namely to the production of more interpretants and representamens. This is Peirce's (1931) unlimited semiosis, which serves as a constant attempt to describe and approach a city. It generates its multiplicity and, in turn, complexity, by producing variables, as well as open-endedness.

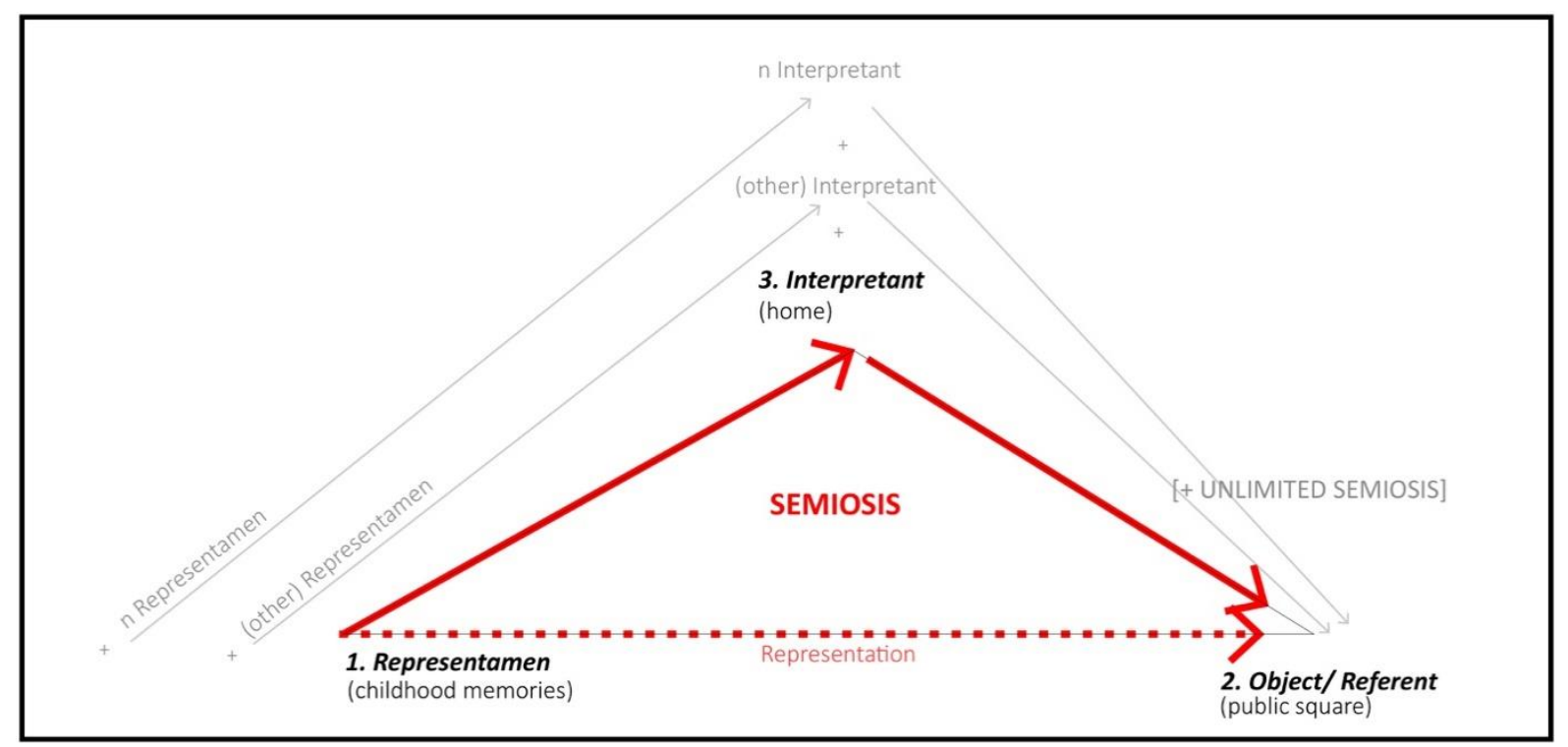

FIGURE 4. Peirce's (1931) semiosis model. Red: operations among three terms' relations. Gray: repetition of process toward unlimited semiosis (visualization: Stamatopoulou, 2020).

\section{Mappings as intra-action processes}

Each of these mappings is made by a subject, within a specific context (social, historical, cultural, etc.). Aligned with thinkers debating the subject-object dichotomy, in this paper reference is made to subjectobject mutual emergence through Barad's (2007) idea of intra-action. Intra-action differs from interaction by denying the pre-existence of the related things, the relata: "It is through specific agential intra-actions that the boundaries and properties of the components of phenomena become deter- 
minate and that particular concepts ... become meaningful" (Barad, 2007, p. 139). For instance, a researcher walks down a street noting impressions. In the intra-action, the researcher and the street emerge as an entity (Figure 5, darker gray area): The "researcher-in-relation-to-the-street" and the "street-in-relation-to-the-researcher."

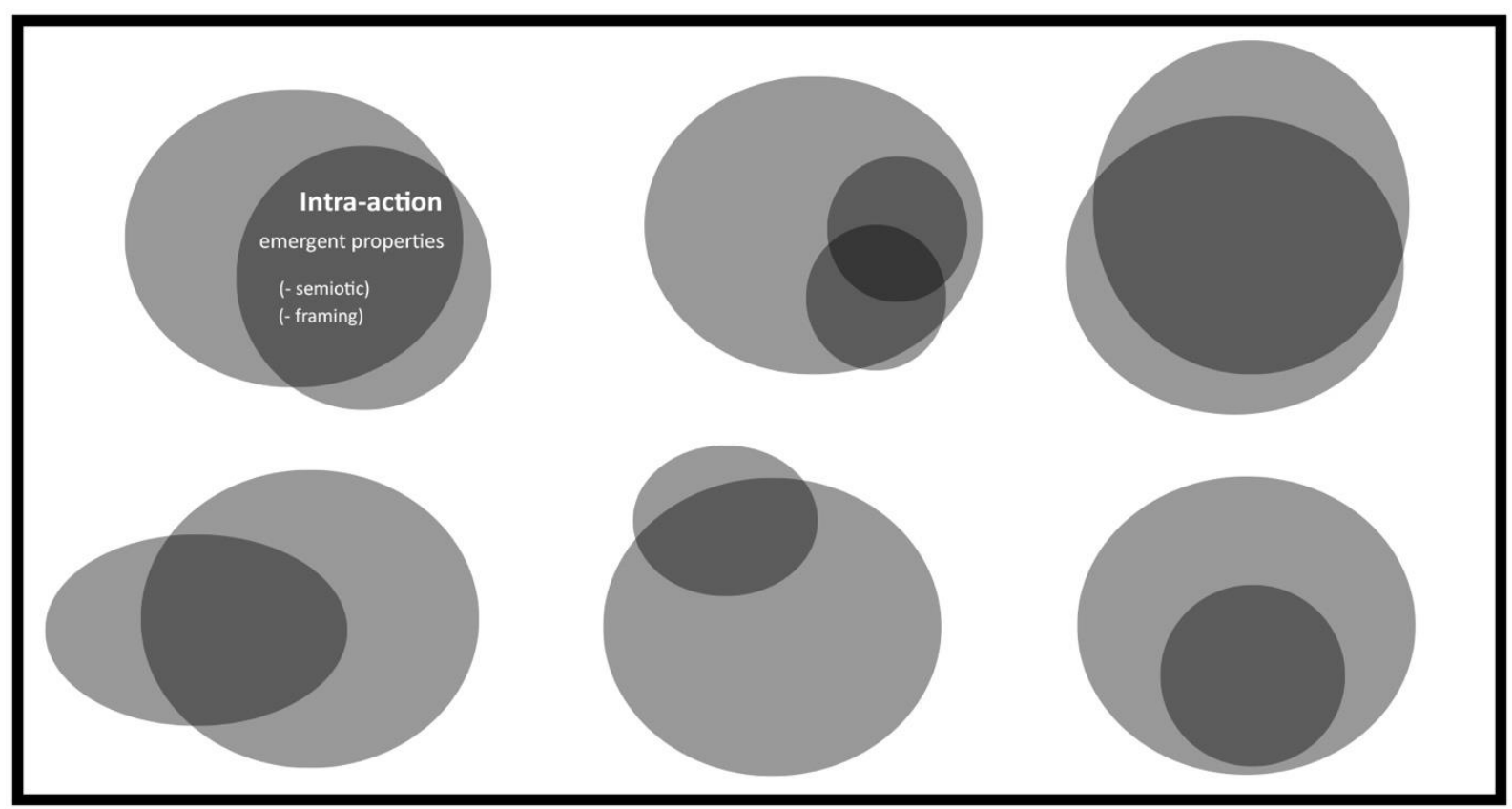

FIGURE 5. Conceptual scheme of how intra-action is interpreted. (visualization: Stamatopoulou, 2020)

Hence, through a mapping as intra-action, the city, the subject, and all emergent properties (DeLanda, 2016) become an entity. ${ }^{6}$ Intra-action sets boundaries. This emergent property is prioritized because it strengthens the conception of borders as not being fixed and therefore under constant negotiation. Cilliers (2001), writing on complex system-environment boundaries, noted that boundaries do not "limit possibilities," but that they are "enabling constraints." Thus, boundaries are frames emerging through intra-actions. Figure 5 depicts variations of intra-actions framings entities (dark gray): In all, one of the two circles is the same thing (i.e. a material field: a public square), and the other corresponds to a different subject.

Regarding the outline of the paper, in a following sub-section, relations with some scientific communities are briefly cited. In the first section a deeper discussion is presented on the methodology's development relating to (1) its schemes; and (2) its capacities explored through the testing of case studies. In a second section a discussion is presented on how these capacities can be translated from the case-specific level into speculations for more generalized and visionary perspectives of relational (urban) design. The paper is closed with thoughts relating to the applicability spectrum of the methodology.

\section{Relations with other communities}

The introduction's references to semiotics, relationality and complexity, mapping and diagram, ${ }^{7}$ and intra-action as well as research by design are linked to broader scientific communities and diverse disciplines. The selection of sources, besides cited reasons, was chosen with the intent to negotiate the transition between theory and practice, and to be involved with experimenting with how concepts and ideas that are developed in different contexts can be translated into practice (Figure 6 , red lines). The in-progress methodology brings the paper in dialogue with mapping and design methodologies. Regarding mapping, the methodologies ${ }^{8}$ developed by architects, designers, artists, and software 
developers are numerous. Considering the paper's targeting, aspects from mapping and design logics are explored selectively with regard to their alliances with the current frame.

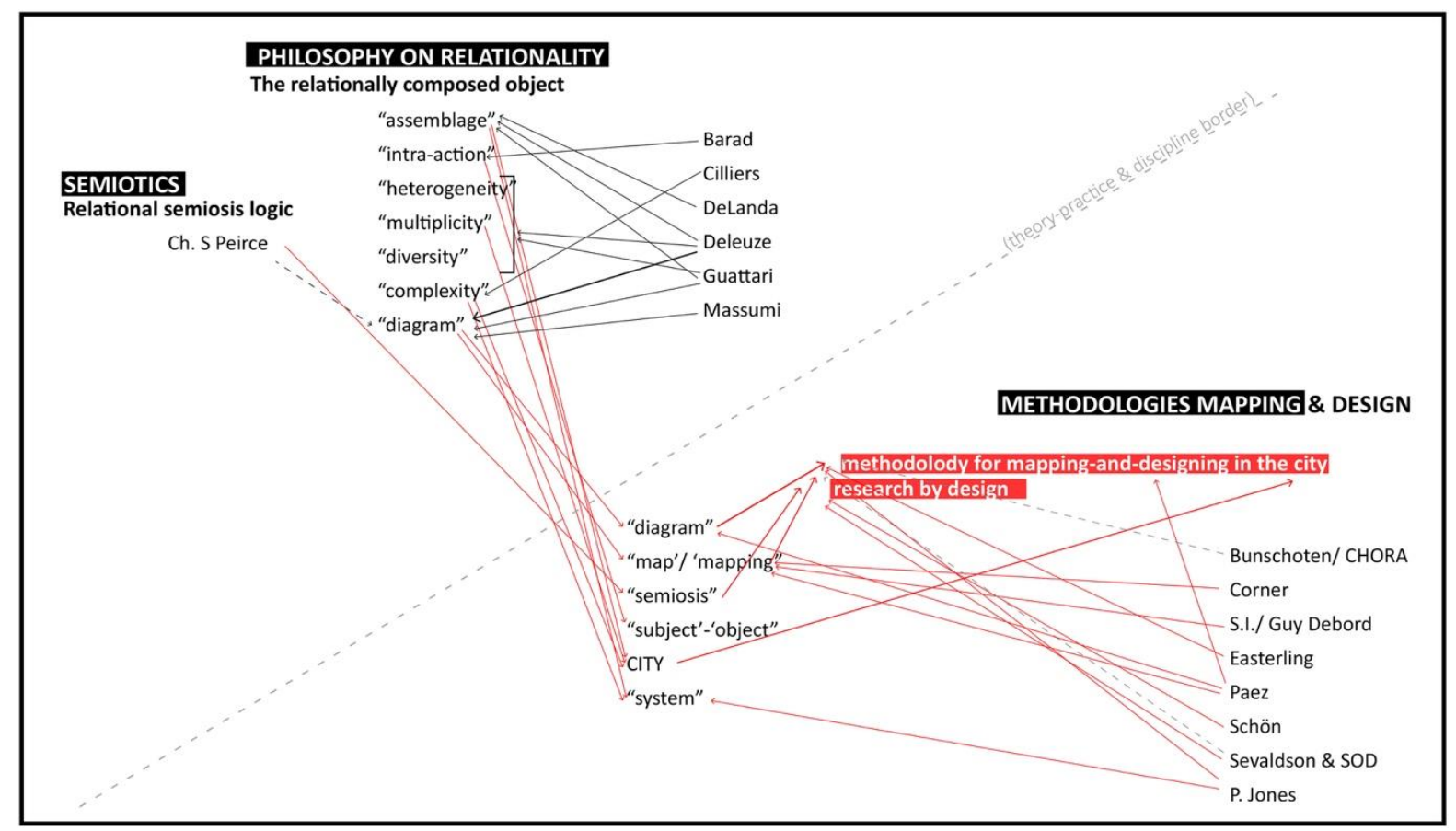

FIGURE 6. Relations with scientific and research communities. Black: How main theories/concepts are used in the paper (introduction). Red: Relations among theory, research by design, and other methodologies. Dashed lines: Possible future influence. (visualization: Stamatopoulou, 2020)

\section{Design by mapping}

James Corner (1999) classified techniques of creative mappings into the drift-dérive, the layering, the gameboard, and the rhizome. The first two constitute influence on the research. Situationists' psychogeographical maps (i.e. Debord's Naked City) negotiate the dominant relations of the physical geography along with their absolute objectivity in a critical way (Paez, 2019). This relates to the reorganizations of the physical (Figure 2) with regard to (the) parameters: For S.I. (1981 [1958]), these are effects of the geographical environment, emotions, and behavior. De-composition and re-composition sequences "cut" the city in pieces and reassemble it. These are (re)framing actions claiming new continuities. The superimposition, operated by diagrammatizing (Deleuze, 1988) of the paper is in dialogue with layering techniques. Corner (1999, p. 235-239) discussed layering through the practices of Peter Eisenman, Bernard Tschumi (Parc de La Villette design), ${ }^{9}$ and Rem Koolaas/OMA (stepwise method for Parc de La Villette proposal). From Eisenman's techniques, in this paper overlapping maps and merge are also extracted, with regard to how they excavate, (as Corner (1999) remarks, non-accessible or nonobvious information, and how they generate design proposals.

The in-progress methodology is in dialogue with Chora's research and practice (Figure 7). Their approach of both research and mapping-based design is of particular interest in this paper. In its abundant practice, design takes the form of strategies, "dynamic masterplans" (Bunschoten, 2003), curating as caretaking (Bunschoten, 2017) and "interactive urban planning" (Bunschoten, 2007; 2017). One of their tools, Urban Gallery, is a stepwise methodology (Bunschoten, 2003, 2005) that consists of four structures. In the first, Mini Scenarios and Operational Fields create, through on-site experiments, the Database. The collection of numerous Mini Scenarios from different locations of a territory ${ }^{10}$ compose an information pool: This identifies engaged actors, problems, and potential for deeper investigation. In the second, the Database evolves into Prototypes. Then the Scenario Games, as "simulation and testing of dynamic environments" (Bunschoten, 2003, p. 60), make the Prototypes 
more precise. In the fourth, the Scenario Games maturate into Action Plans, in which the Prototypes are adapted and implemented. Additional highlighted features of Chora's practices are the integration of multi-scalarity within the same application as well as intertwining ${ }^{11}$ and interaction operations, toward newness ${ }^{12}$ - birth in the system (Bunschoten, 2003, p. 61).

\section{On designing}

Keller Easterling's thoughts on active form (Easterling, 2014) are a resource for the speculation of diverse design logics (Figure 7). The activity of a form lies in its capability of interacting within the system where it is located, and of affecting it. An active form is like "spatial software," or "time-released protocols that generate or manage a stream of objects and spaces" (Easterling, 2014, p. 39). It also functions as a spatial lever, which might be, among other things, a multiplier (contagious detail causing repetitions), a remote control (distant event affects a local condition), or governor (protocol for growth or interdependencies in the larger urban calculus). Therefore, active form can be seen as a broader design logic targeting to systems of relations. This becomes clearer in Easterling's (2017) Medium Design, in which design performs as the "managing of potentials and relationships between objects." For Easterling (2017), Medium Design reveals more information and increased difference, linked to the relations' heterogeneity and the complexity that the paper pursues.

Discussion about design methodologies touches the broad field of design methods. ${ }^{13}$ From this, the paper is inspired by Systemic Design principles (Jones, 2014, 2018) ${ }^{14}$, as also shown in Figure 7:

(1) In Idealization, an "ideal state or set of conditions ... towards a desirable outcome" (Jones 2014: 108 ) is formulated. Driven by a vision, it might serve as future-finding process through "multiple alternative scenarios."

(2) In Appreciating Complexity, the comprehension of "the dynamic complexity of multi-causal" (Jones, 2014, p. 109) "wicked problems" (Rittel and Weber, 1973) is stressed.

(3) Purpose Finding makes clear how purposes get defined by agreement in dynamic ways that keep them open to re-design(s).

(4) Boundary Framing, ${ }^{15}$ associated with problem framing, aims at "the most effective fit between a concept and its target environment" (Jones, 2014, p. 111) through iterative processes.

(5) Requisite Variety controls the functional complexity of a design, so that it is able to "provide sufficient options for interacting with ... its target environment's complexity" (Jones, 2018, p. 35).

(6) Feedback Coordination (design) has to do with the integration of critical feedback relationships into the design process. Feedback undertakes the "coordination of a system's dynamic fit to environmental and contextual functions" (Jones, 2018, p. 35).

(7) System Ordering is the organization of a system's elements and their relationships.

(8) Generative Emergence integrates the creativity of emergence into design in two ways: by Compositional Emergence, which "manifests in design activity as an outcome of ordering or the construction of artificial micro-systems for adapting an artefact to environments" (Jones, 2018, p. 35); and by Created Emergence, that manifests from organizing systems.

(9) The Continuous Adaptation, from the phases of development and design to deployment and operation, "maintains the preferred system purpose and objectives throughout the lifecycle of adaptation, the conformance to environmental demands, and related system changes" (Jones, 2014, p. 109).

(10)Self-Organizing, linked to Generative Emergence, embeds actions of awareness, incentives, and social motivations. These, by designing conditions, "accelerate organizing behaviors" (Jones, 2014, p. 120).

Systems Oriented Design (SOD) and Sevaldson's work on research by design are broadly of the same field to which this research frame relates, although in a dialogue that is not (yet) obvious (i.e. GigaMapping ${ }^{16}$ methodology [Sevaldson, 2011]). Multi-perspective analysis and design (Sevaldson, 2018a) 
is an intention shared, particularly through the paper's objective for multi-parametric approaches. More familiar with the current phase of the methodology development is the ZIP analysis method (Sevaldson, 2017) in which "Z" stands for zoom into areas/points for deeper analysis; "P" stands for potential or problem, and "I" stands for innovation, intervention, and idea.

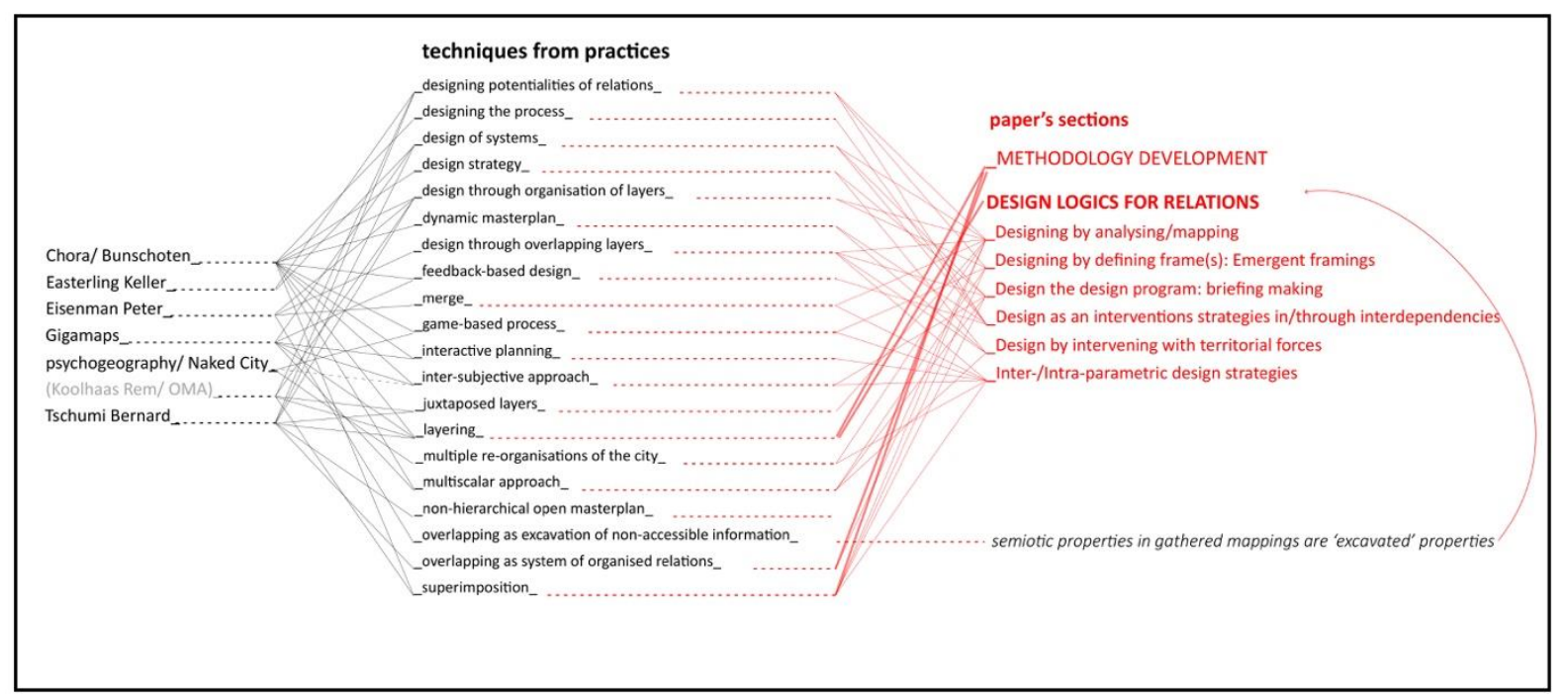

FIGURE 7. Relations (in red) among extracted techniques (as items of logic) from detected practices (on the left) and the speculations for design logic (on the right). (visualization: Stamatopoulou, 2020)

\section{METHODOLOGY}

\section{The methodology's development scheme}

The methodology evolves gradually through research by design (Sevaldson, 2010). Its development scheme (Figure 8) is founded on the logic of the triadic relational model (Peirce 1931). By embedding unlimited semiosis (Peirce 1931), use of the model enforces the continuity of a process, encouraging conceptions of an object as open and dynamic. ${ }^{19}$ Apart from the city, such an object is the in-progress methodology - the research.

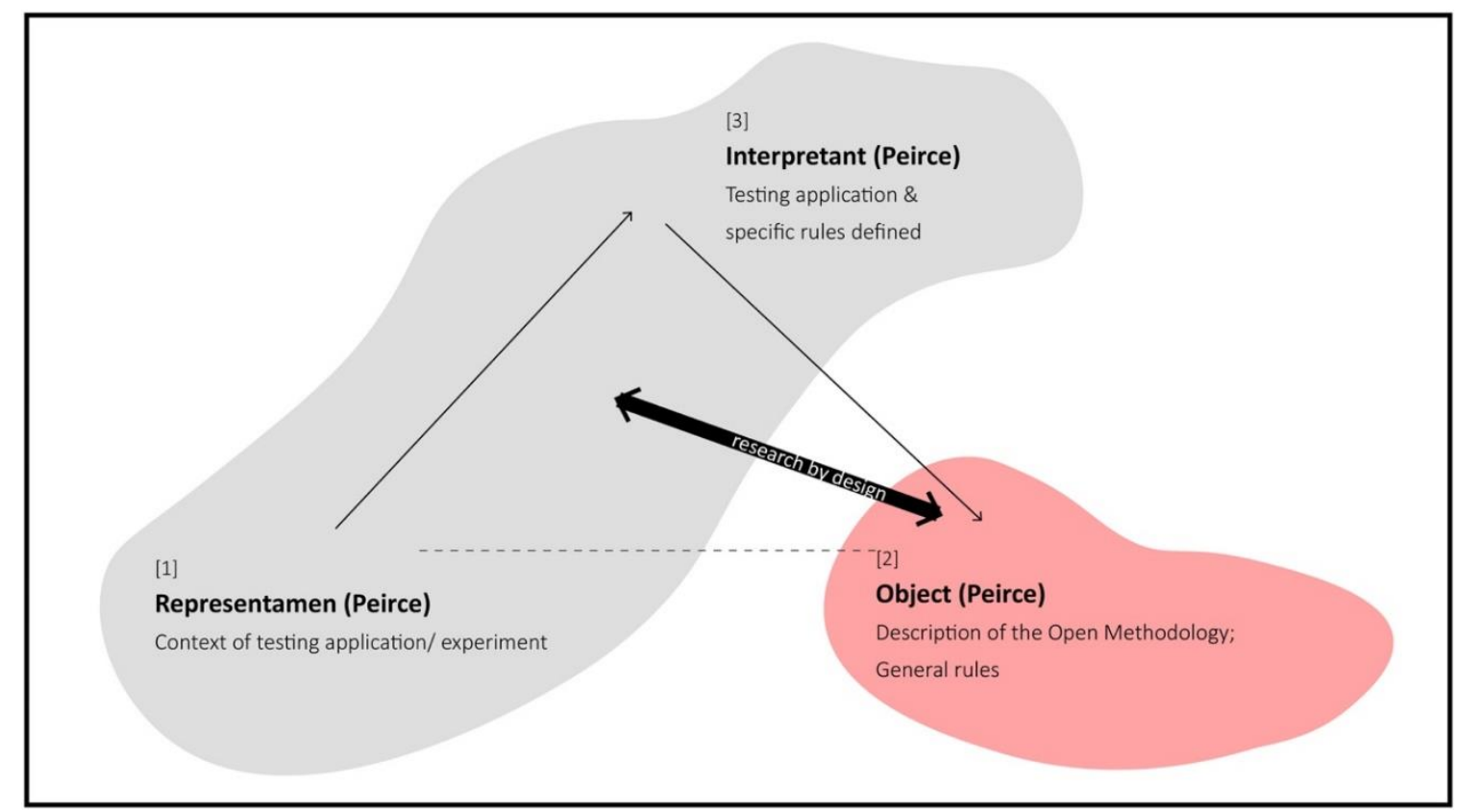

FIGURE 8. Peirce's relational model, applied for the development of the methodology (visualization: Stamatopoulou, 2020). 
The methodology (Figure 10) is further organized by three interdependent levels of action: In the first, data are gathered; in the second, relations are organized among them; in the third, the logic is tested through specific case studies, operating design in the research by design. As depicted in Figure 8, the methodology's general level (colored red), namely the research in the research by design, becomes the second term (object) of Peirce's triangle. Each testing application (Figure 8, gray area) functions as the relationship between the first and third terms of Peirce's model. This relationship informs the research each time. The context of each test, regarding its properties as an experiment (i.e. territorial scale, type of content), corresponds to the triangle's second term (representamen). The way the testing application manifests constitutes Peirce's third term (interpretant). Such organization for the methodology's development intensifies the key role of the third level.

The tests are expected to be multiple. Thus, the methodology becomes open to Peirce's (1931) logic of unlimited semiosis: through its dialogical performativity, the definition of the methodology's general rules is continuously (in)formed. This strengthens the intended openness for the methodology's development: The red area in Figure 8 takes input by many tests (gray areas). These relations of shared information are relations of feedback. Figure 9 makes clearer how the gradual development of the methodology (red areas) is based on multiple feedback transitions between specific and general, namely between design (as experimental practice) and the research it serves. Feedback relation advise the system with regard to its expected performance, and consequently might indicate changes: These, through the lens of un-limitedness, can be variations of the methodology. This happens, for example, in Figure 9, through a differentiated actualization of the same experiment. Hence, feedback does not necessarily trace correct/faulty things, but generates variations, maturity, and newness.

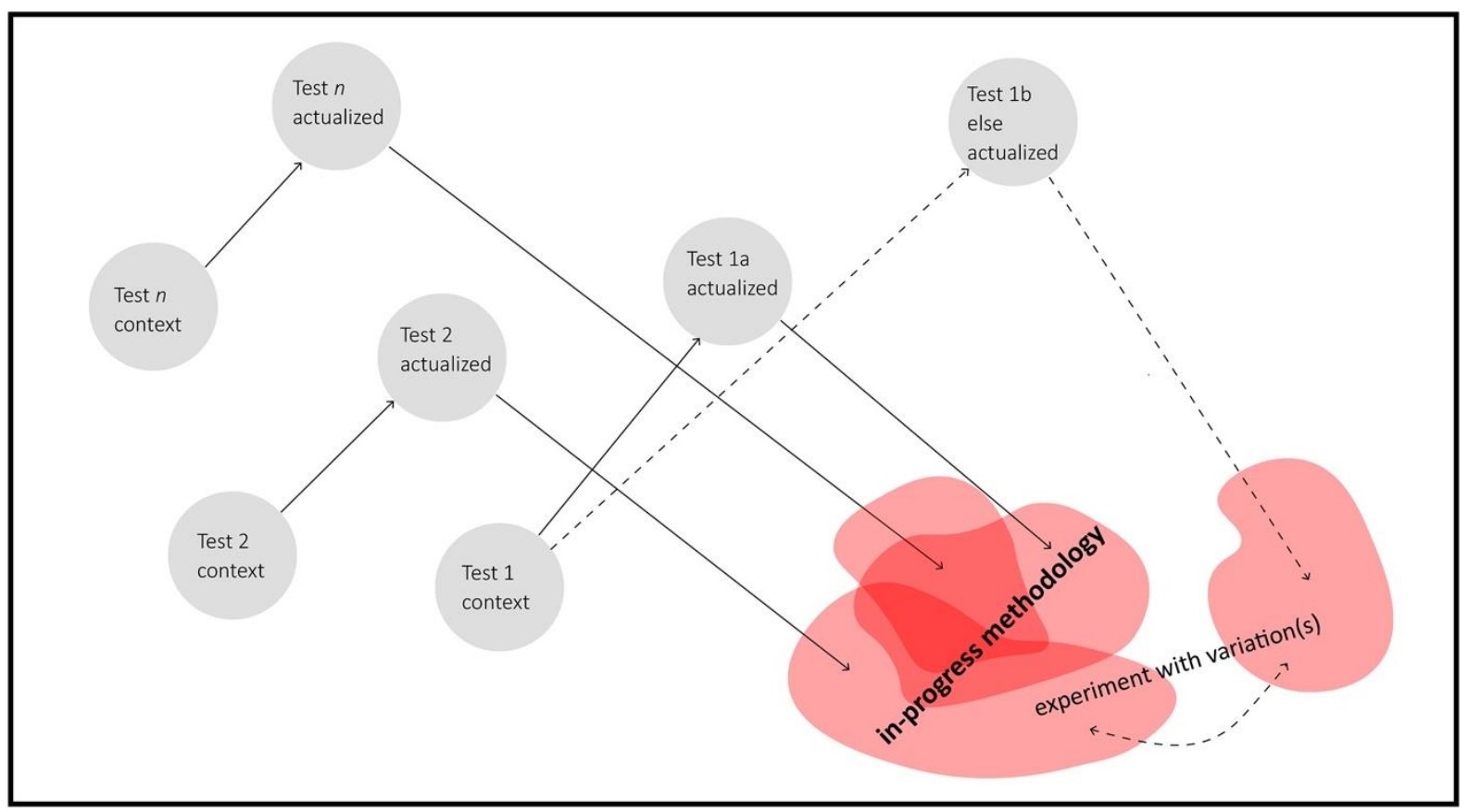

FIGURE 9. Visualization of the methodology's gradual development. The repetition of Test 1 , as a different manifestation, produces variation, depicted as shift. (visualization: Stamatopoulou, 2020)

\section{The methodology's scheme}

The methodology's scheme (Figure 10), composed of three levels (red), is explored and analyzed through the combinatory consideration (red arrows) of two testing case studies (gray). In the first level (Figure 10), diverse, distinct mappings by different subjects are gathered (1) by triggering mappings (i.e. through fieldwork research, workshops); (2) by collecting existing ones; or (3) by combining both ways. 


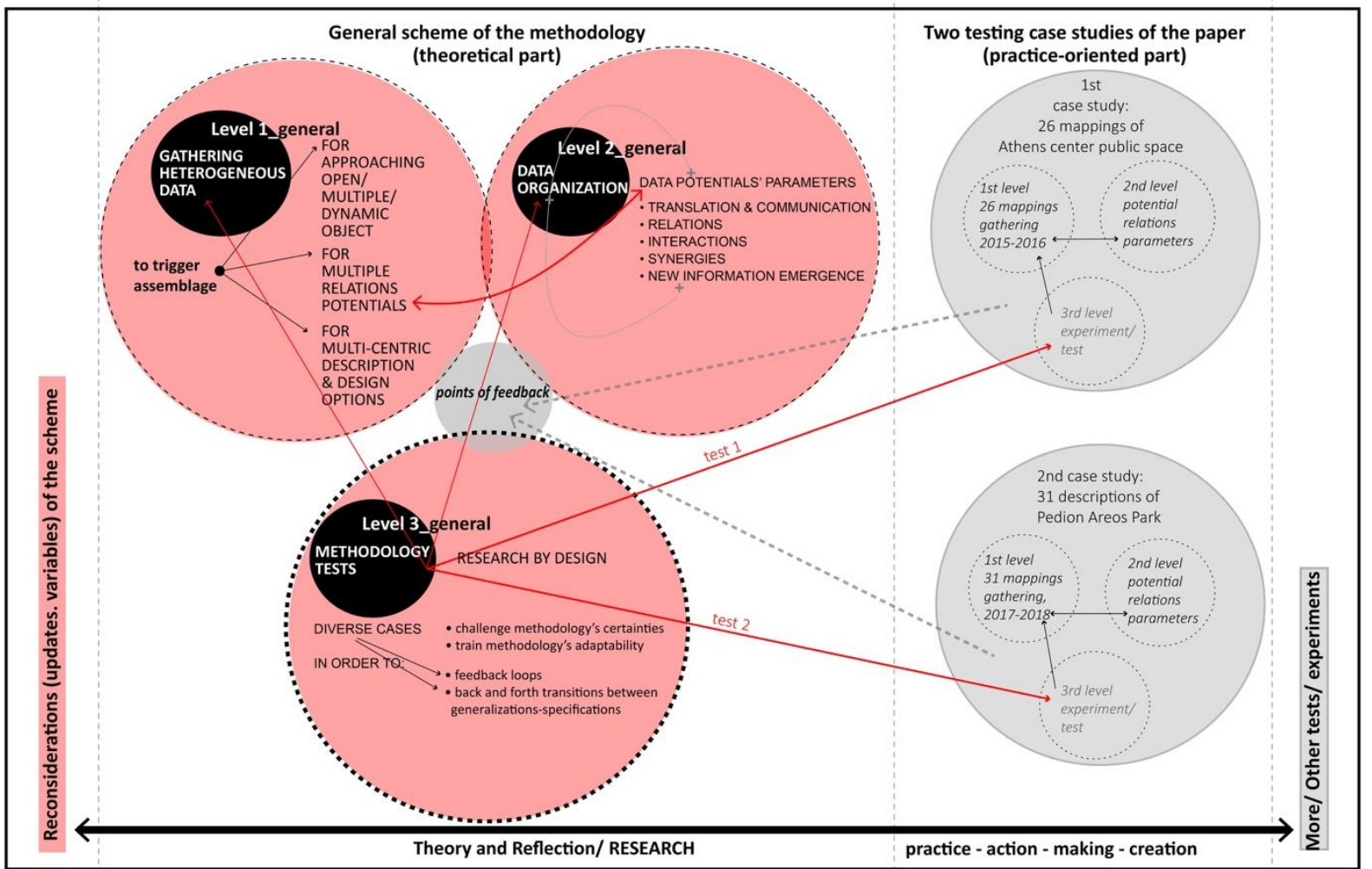

FIGURE 10. Methodology's scheme in detail, integrating paper's tests (gray circles on the right). Coloring refers to the notations of the more abstract visualizations in Figures 8 and 9. (visualization: Stamatopoulou, 2020)

The way of gathering influences the control and guidance of the outcome, and in turn, emergence potentials. Control cannot be completely avoided, but it can be reduced. For this reason, with gathering, emphasis is on the diversity of the mappings' format and given guidance is minimized by avoiding protocols. Not aiming at statistics, the gathering follows a sampling logic, under the criterion of heterogeneity and multiplicity, assuming that it gives access to greater complexity, more information, and more potential. This cultivates perspectives for the appreciating complexity and requisite variety principles of systemic design (Jones, 2014). Heterogeneity begins from subjects' diversity, that through Barad's (2007) intra-action, matches to:

(1) diversity and multiplicity of objects, namely cities to be revealed;

(2) multiplicity of agencies;

(3) diversity of system-environment boundaries/framings, that expands to

(4) diverse scales of approach: The different approaches are diverse relations with the mapped territory.

These vary from relations with it, i.e. through Google Maps (mediated ways) to on-site mappings (less mediated ways, like in psychogeographical maps). Heterogeneity also corresponds to an expected widened spectrum of unveiled properties and capacities: Properties concern "what an entity is," while capacities concern "what it can do" (DeLanda, 2016, p. 52). For example, a mapping of a park might mention existing properties (i.e. seating infrastructure) and express visions through detected potentialities or latencies (i.e, vividness potential, through integration of more activities in the park). The conception of the capacity can be amplified through the potential problem in the ZIP analysis method (Sevaldson, 2017). 


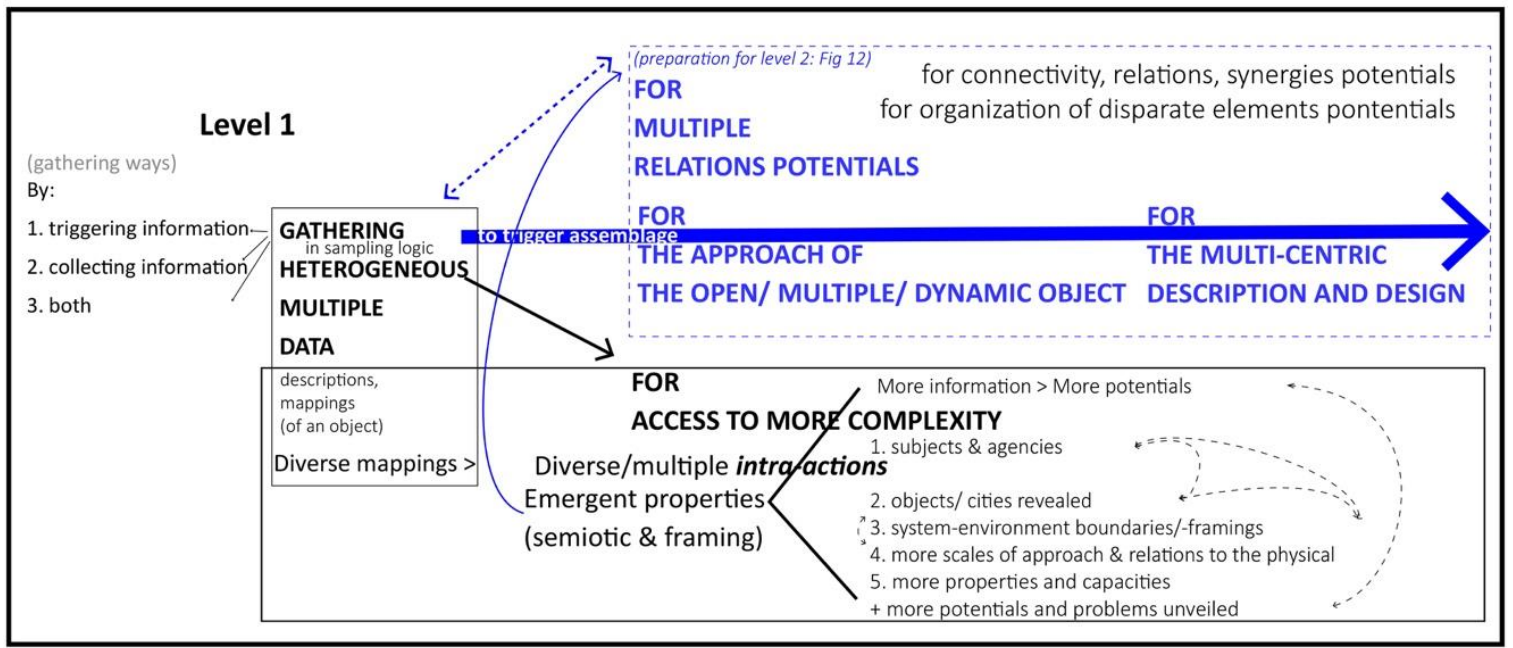

FIGURE 11. Main terms and relations for the data and information gathering (visualization: Stamatopoulou, 2020).

Furthermore, the heterogeneity-multiplicity criterion is prioritized, because as a prerequisite of assemblages (Deleuze and Guattari, 1987), it allows one to "bring together disparate elements and organize them," and to "connect, link up, create relations between terms and objects of differing nature" (Zdebik, 2012, p. 26). Considering the interdependence between the first and second level, heterogeneity facilitates the organization of information (second level). Therefore, the first two levels may evolve together. At the same time, heterogeneity affects design options, as it enforces multiperspective approaches by embedding diverse parameters and involving inter-subjectivity.

The second level (Figure 12), close to Easterling's (2017) Medium Design, is used to investigate potential relations and synergies among gathered mappings. By analyzing ${ }^{17}$ their information, in this level ${ }^{18}$ translation, communication, and interrelation parameters are defined among them, under the criterion of heterogeneity. Parameters are crucial for system ordering (Jones, 2014) options and for revealing networks beyond the physical (either in a single mapping or in the system of mappings). Communication refers to relations, which target potential interactions and synergies. More analytically, in this level, each mapping is not considered (only) as an independent one. Essentially, its information is not limited to its context. Each mapping is part of the system composed by the organized, gathered mappings. Therefore, through this level, the methodology goes beyond the contexts and contents of its gathered mappings, through the connections (indicated by parameters) among their initial information. For instance, a mapping connects certain locations in a neighborhood. In the mapping's system, its value is not limited to that, but it expands through its synergies with other ones. This way, new properties and capacities (DeLanda, 2016) as well as new information might emerge (Figure 13, in red). Such emergence is performed through superimposition operations.

\section{DATA ORGANIZATION}

\section{Level 2}

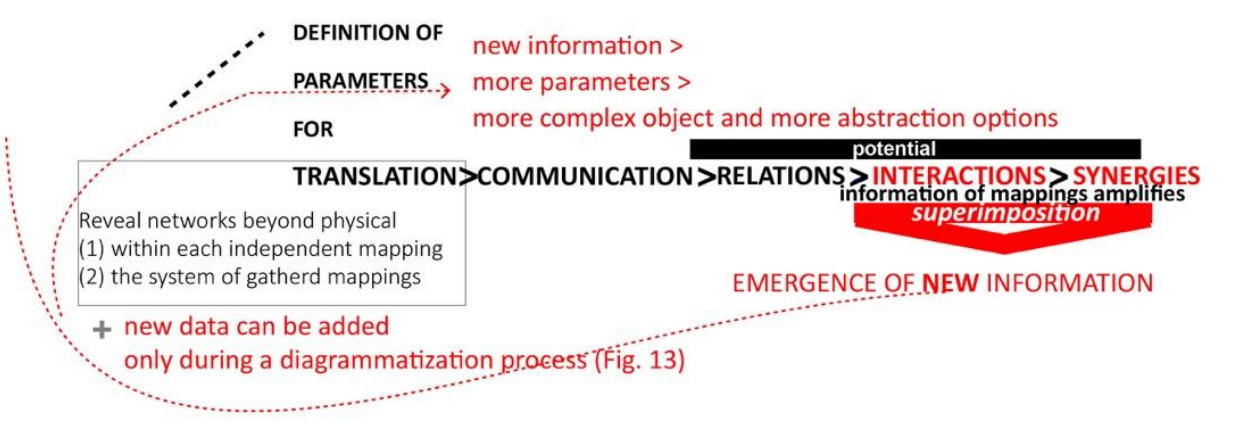

FIGURE 12. Scheme of the second level of the methodology (visualization: Stamatopoulou, 2020). 


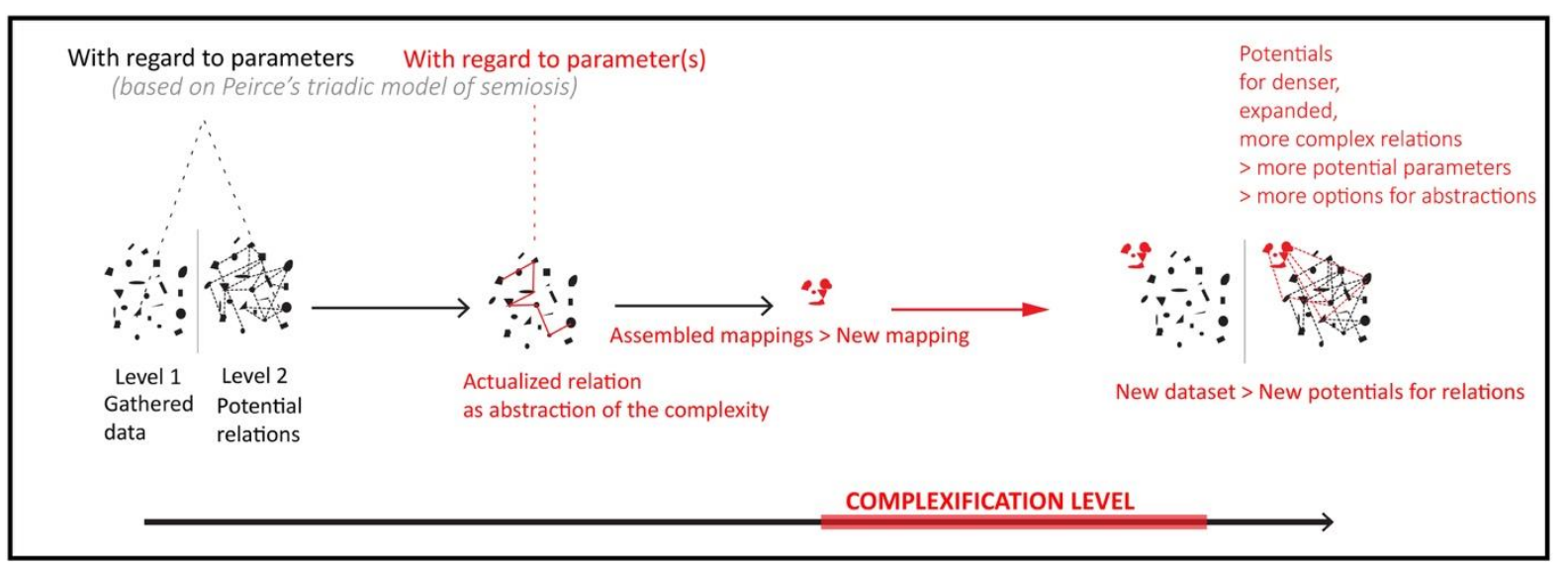

FIGURE 13. Scheme of how data are related and how complexification perspective emerges (visualization: Stamatopoulou, 2020).

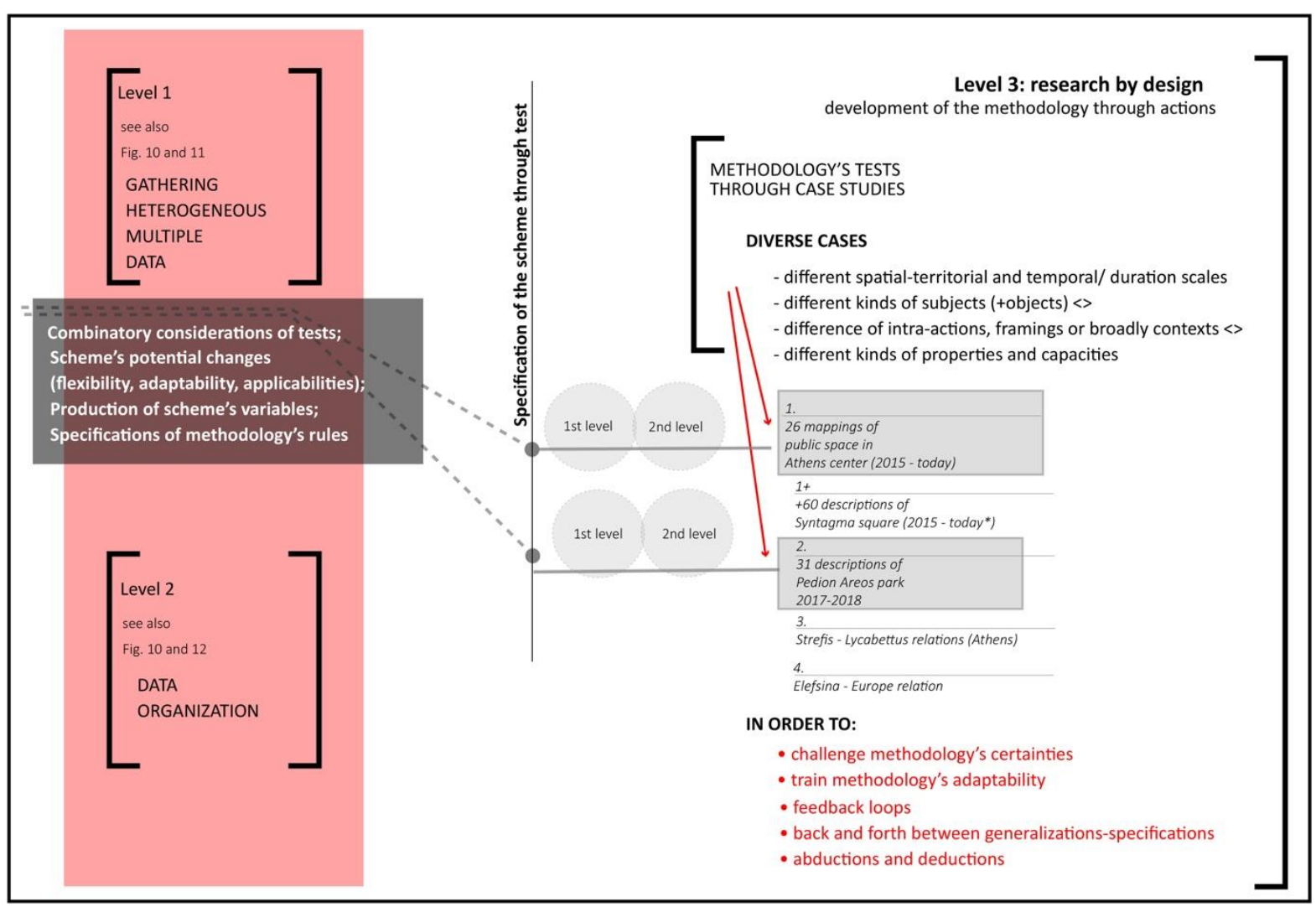

FIGURE 14. The third level of the methodology's scheme and its relations with the other levels (previous Figures). Red-gray coloring matches to the general-specific as in previous figures. (visualization: Stamatopoulou, 2020)

The third level (Figure 14) keeps the methodology's logic open, in terms of flexibility, adaptability, updatability, and variability, through the experiments (Figure 9). These only serve the research for the development of the methodology, so they are non-exhaustive tests in varying, specific cases. The tests' diversity challenges the methodology's certainties on different grounds, to train its adaptability and cultivate its applicability to different contexts. The multiple tests also concern the independence of the methodology from the specific features of a testing case: The features that can be general for the method become divisible from features determined by a case. The detectability of these relations' boundaries is intensified when seeing them comparatively; this enhances feedback loops, yet it 
transitions back and forth between generalizations and specifications, negotiating abductions and deductions.

In this work, this is attempted by assessing the methodology through a combinatory consideration of two testing applications in the city, conducted independently in previous phases of the author's research. If each test contributes to the development of the methodology (Figures 8, 9), then, their combinatory views, and their dialogical criticality, may give stronger feedback in their intersections.

\section{Visualization of the methodology into an Interactive Open Map}

Heretofore, the organization of the mappings and their relations are visualized through an Interactive Open Map (IOM): this is influenced by relational databases and GIS logic. The visualization presented here is simple and manually made, ${ }^{19}$ functioning as support for reflection and research. These actions are aimed at describing how an improved future visualization might operate, at defining the rules of such a system, and at exploring its potential capacities as a design interface. IOM is the system of a database, a table, and a map (Figure 15).

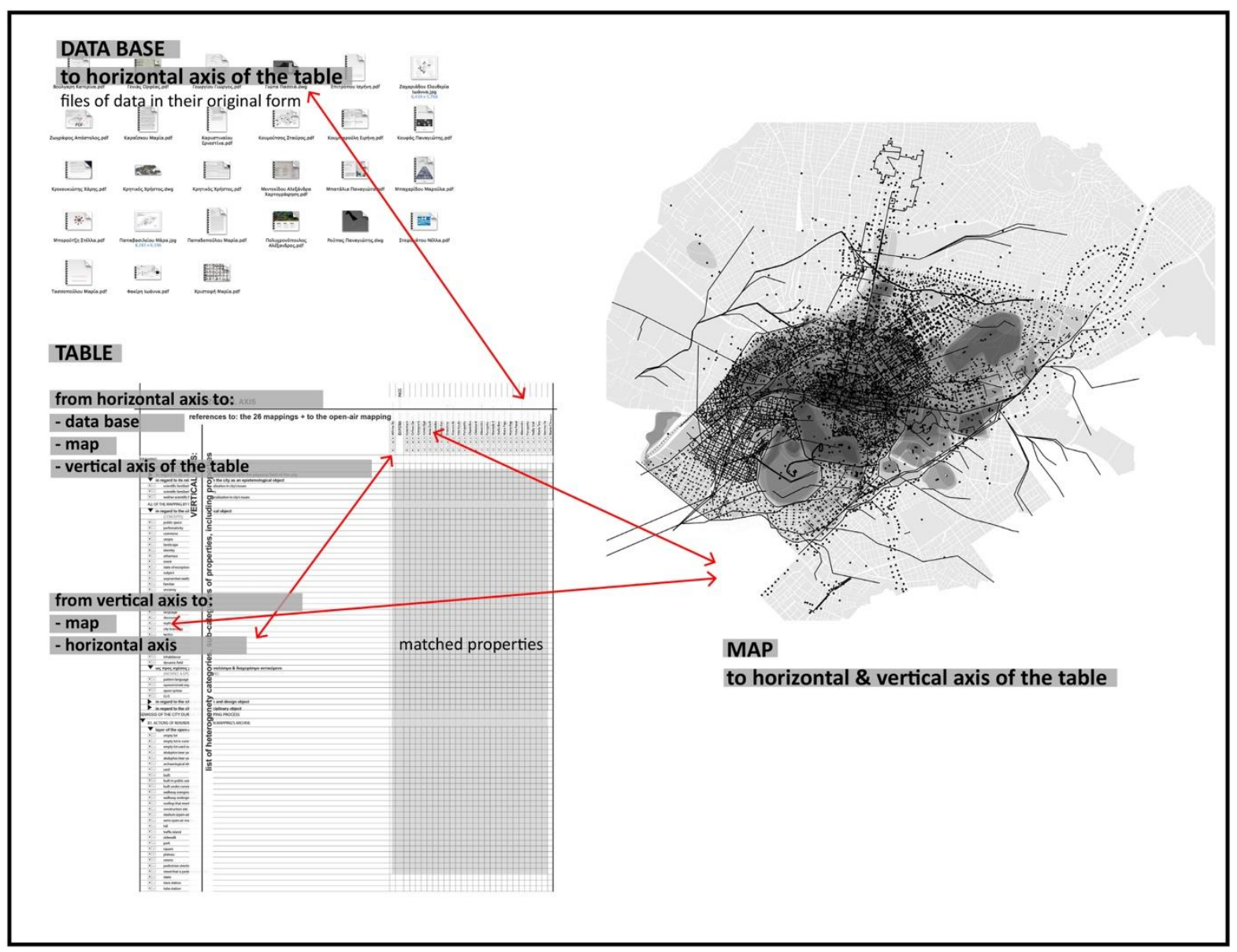

FIGURE 15. Selection options (red arrows) between the database, the table, and the map in the IOM (visualization: Stamatopoulou, 2018).

The database contains the gathered mappings in their original form. The horizontal axis of the table integrates references to these mappings, and the vertical axis to the properties' list as translated and organized in categories. In the table's cells, the properties of the cited mappings are matched. On the map, the references to physical locations are codified and noted: Each black dot corresponds to the 
reference of a specific location; each black line corresponds to paths; and the black, irregular schemes to abstract references to areas (Figure 16, later).

The database, table, and map are supposed to be interconnected through options for selections (Figure 15: red arrows). With some of these:

- The database is accessible from the horizontal axis of the table. For instance, selecting a mapping there gives access to the original mapping. This permits one to examine, at the same time, the translated-codified form of a mapping (in the table-map relation) and its original one, as made by its subject. This option bridges the division between top-down and bottom-up approaches: An original mapping in the database might be a bottom-up approach, while its translation into an IOM shows its top-down look.

- The map is accessible from both axes of the table (Figure 21).

- Selections on the map reference both axes of the table (Figure 23).

Combinations of selections among the IOM's elements are also possible: this increases the potentials of diagrammatization, fueling the system's generativity (Figure 13). Conceiving mappings as intraactions, makes the relations between organized information/properties (table) and the material field (map) in the IOM, crucial. This table-map relationship delivers the challenge of going beyond the physical without getting detached from it.

\section{EXPLORING THE METHODOLOGY THROUGH THE CASE STUDIES}

The methodology's development contains experiments in diverse cases of territories. The phase presented in the paper is the first attempt to make a combinatory examination, enriching speculations about transitions from data to design.

The first case study is an experiment of 26 mappings, by 26 subjects, of public space in Athens Center, carried out as an exercise in the context of a postgraduate course in 2015. Each subject was asked to map a public space with regard to its research interests, without any guidance for collaboration with the other subjects. Through the independent mappings of the subjects, more layering, as well as de-composition and re-composition options are possible. No other constraints (i.e. territorial boundaries, topics to consider, mapping format) were given. Additionally, all of that had to stay tuned in Athens Center and public space. The subjects of the mappings have converging scientific backgrounds (architecture, design, arts, social sciences), but their methods are quite diverse.

Parametrization is decided within each case study context. For this, a scenario is formulated: Emphasis is given on the assumption that every mapping is a semiosis process. Specifically, every mapping is being framed by (a) its subject (i.e. concepts ${ }^{20}$ through which the subject approaches the city); and (b) whatever frames it (i.e. subject's cultural context, role, discipline). These properties, organized into sub-categories (Figure 16), belong to the broad category, "A Framework of the Mapping." In parallel, the subject frames the city, when mapping, or else intra-acting with it. These properties compose the category, "B Semiosis during Mapping Process."

More analytically, the sub-categories of "A Framework of the Mapping" concern things (A1) framing the subject of the mapping and (A2) things framing the mapping process. The latter are further defined as relations of the mapping process with the city; the city as an ideological object, as an object of analysis, as an object of intentional research, and as an interdisciplinary object. This way, it embeds more detailed properties that contain theories and tools of approach (i.e. Space Syntax, Alexander's Pattern Language), as well as combinations of divisible disciplines (i.e. mathematics, biology). In category B, all sub-categories include properties related to the operations during the mapping, including how each mapping refers to the city. Some categories of properties are related (Figure 16: in red arrows), and some properties might belong to more than one category. Apart from organizing the potential for relations, the definition of properties within general categories intends to form different degrees or variations. For instance, the number of concepts and theories (not shown in Figure 16) used in a mapping may define degrees of intentional framing of the mapping. Such a degree contributes to the degrees of a mapping's openness, as these are further determined by other properties. This is the 
case, for example, if the mapping is in an editable format (degree of editability), or if it can be read in more ways (degree of representation's hypertextuality). In this scenario, setting degrees enables parameters to function as qualitative measurements of the mappings and of the mapped/(possibly) signified city. This cultivates the horizon for the intended merging of quantities and qualities.

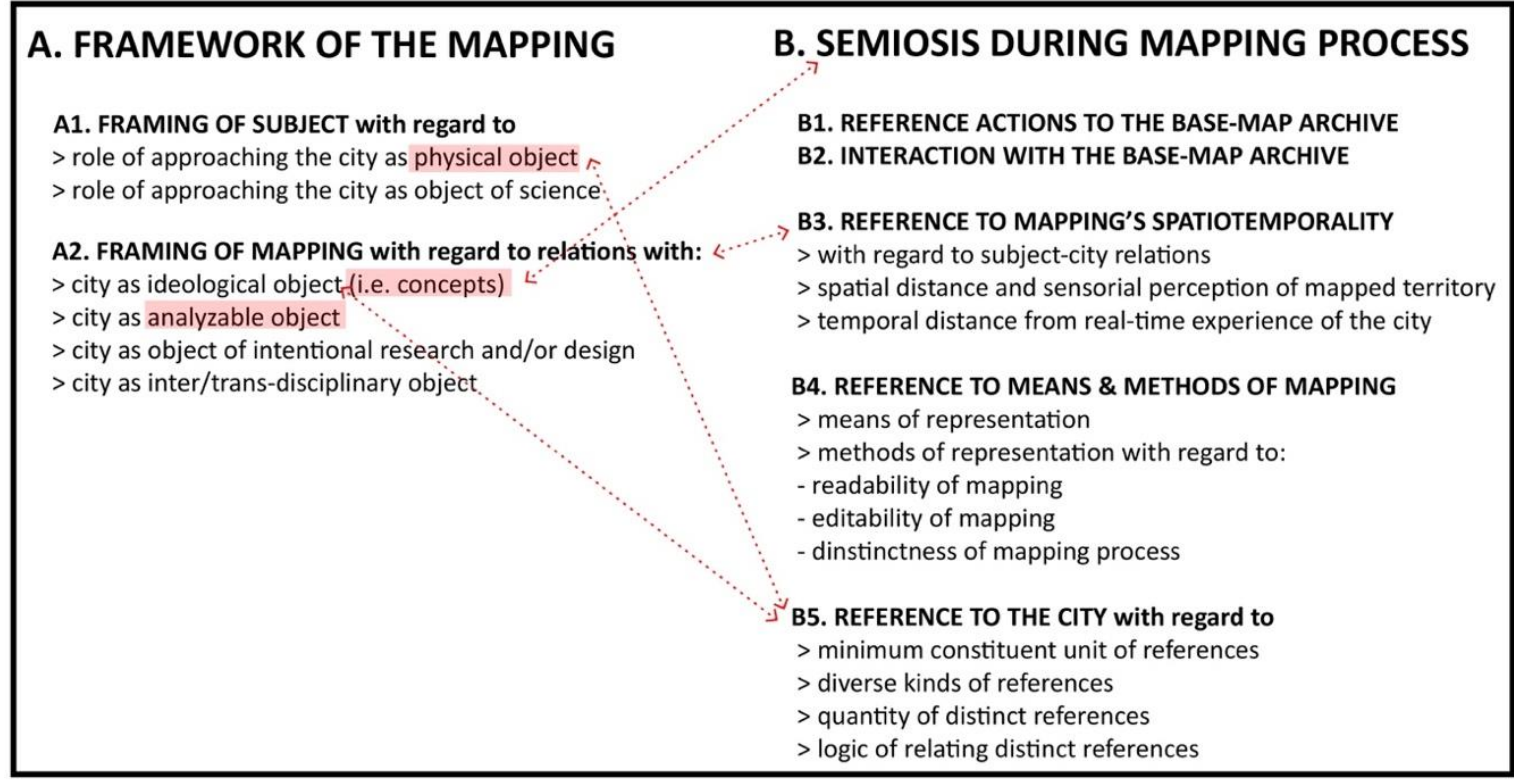

FIGURE 16. Properties classification for the first case study into two general (A) and (B) categories and sub-categories of properties. Red arrows indicate potential relations of properties. (visualization: Stamatopoulou, 2020)

In Figure 17, some of the gathered mappings are shown in their original form, unveiling the multiplicity of the same thing. These manifestations, despite their visualization in the so-called top-down appearance, correspond to very different relations with the physical terrain.

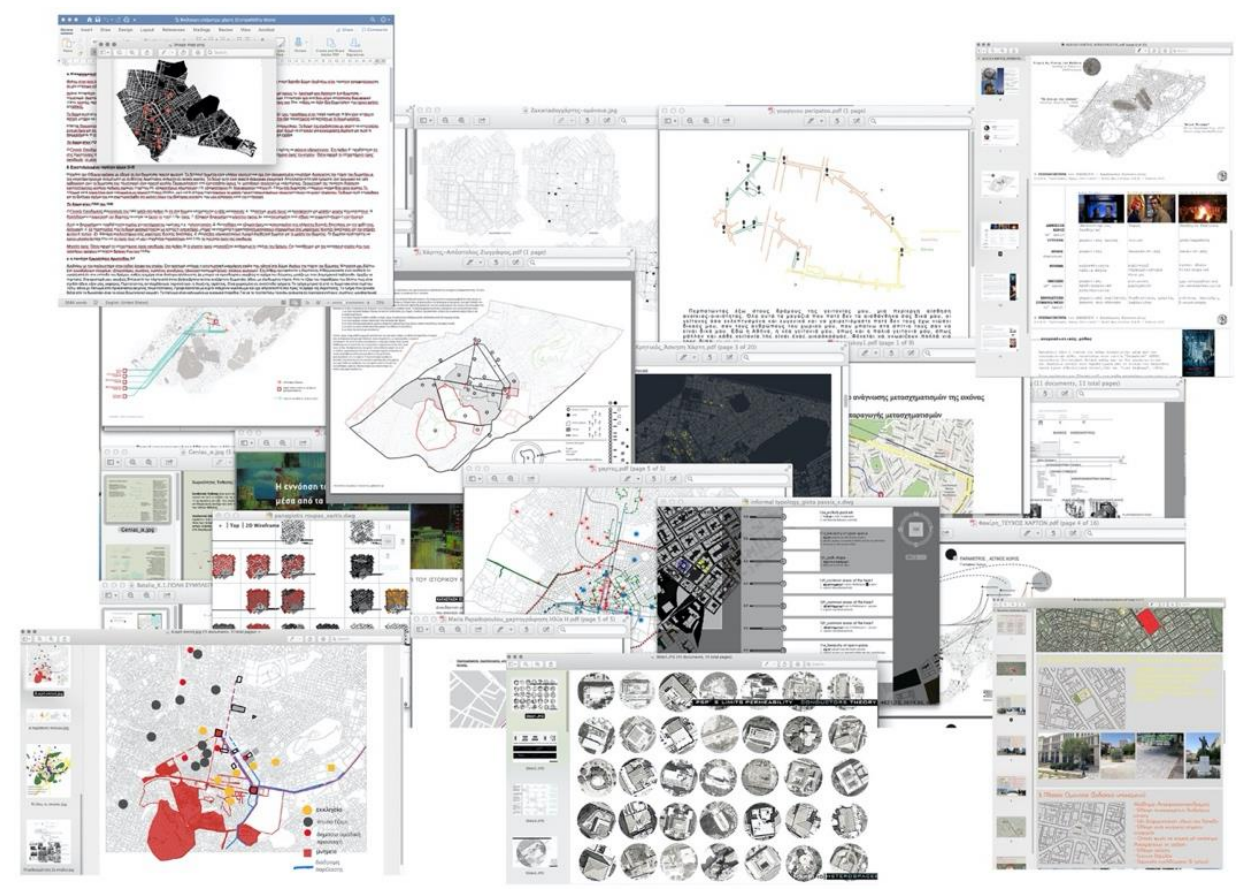

FIGURE 17. Sample screenshot of some of the 26 mappings in their original form (visualization: Stamatopoulou, 2016) 
For example, there are few mappings based on lived experience. Although this detail is not directly visible in their maps, it is detectable in their accompanying texts, which are integral parts of the mappings. This intensifies the value of the mappings' multimodality. Furthermore, it confirms the significance of integrating properties of the mappings' methods and processes during parametrization.

In Figure 18, each map shows the codified references of each gathered mapping to the city. Each mapping is a different intra-action, which frames the city in a different way. The spatial manifestation of this framing is observable in the codified form of each mapping. In Figure 18, this manifestation concerns the black notation of the references within the marked boundaries. These maps are argued as different versions of Athens Center public space, as different cities, beyond and within the same city. Moreover, Figure 18 is a visual explanation of how the in-progress methodology sets boundaries for approaching a physical territory. The field is not pre-cut into pieces and distributed to subjects for zooming in, like partitioning in Urban Gallery (Bunschoten, 2003). Each mapping, as framing, is responsible for cutting the territory, to find the pieces that (re)organize its city.

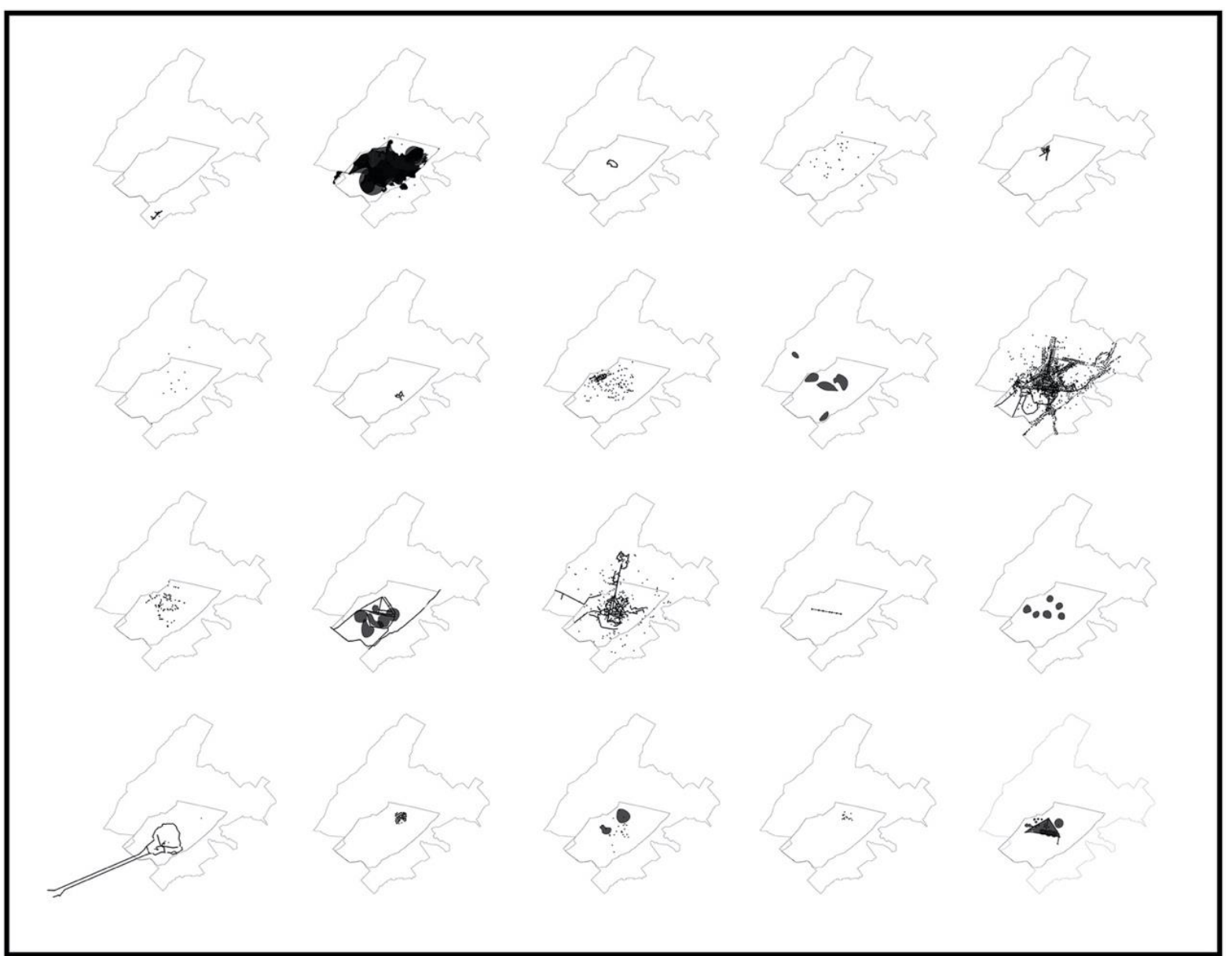

FIGURE 18. The 26 mappings in their codified form. Outer shape: Athens municipality borders; inner shape: borders of initial base map (broader area of Athens Center). (visualization: Stamatopoulou, 2016)

This gives access to the boundary resulting from all the mappings and, thus, the framings' superimposition, as depicted in the map of Figure 19. In the first testing case, this boundary expands the initial boundary of the base map (Figure 19: inner borderline; Figure 19: red line), as well as the formal boundaries of Athens municipality (Figure 19: outer line). Additionally, the overlapped boundaries embed the different properties of the mappings to which they are related. This means that the boundaries have properties, which are overlapped as well. By this, the emerging boundary is not only expanded but also densified. Its density not only concerns the geographical information arising from the connection of diverse networks, but also the overlapping of multiple properties. 


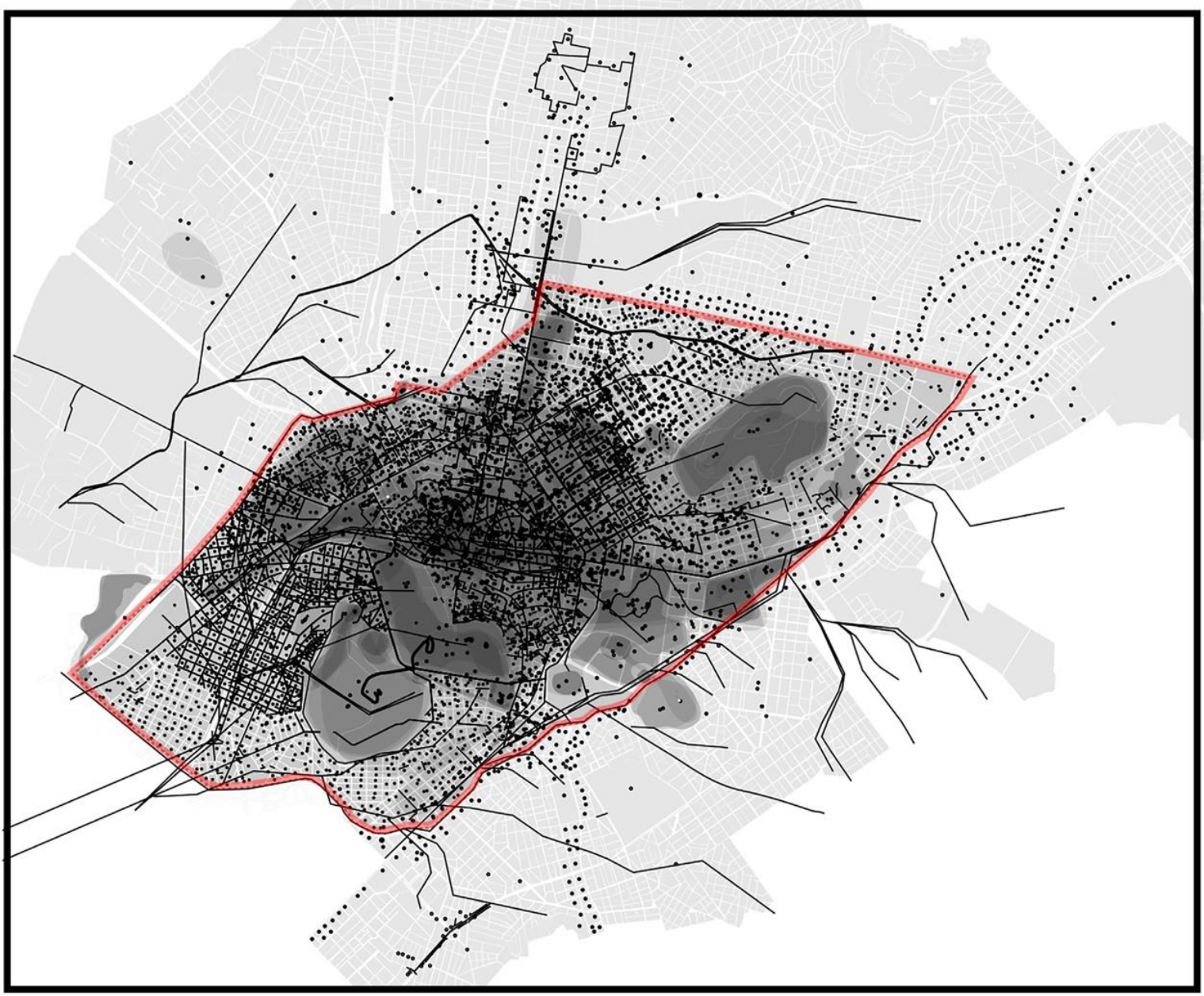

FIGURE 19. The overlapping version of the 26 mappings in their codified form: dots are references to specific locations, lines are references to paths, and irregular schemes in opacity are more abstract references to areas of the city. (visualization: Stamatopoulou, 2016)

The second case study is a testing application in Pedion Areos Park in Athens Center. Thirty-one mappings, in the form of descriptions with verbal content, were gathered in a sampling logic, prioritizing heterogeneity. This test differed from the first one, with regard to the following parameters, which feed observations of complexity:

(1) Data gathering

(2) Types of data: Gathering combines triggering mappings and collecting existing ones. Collection draws from published discourse, literature, and poetry, inserting different kinds of representations. The triggering of mappings further differs, because it was made by the author on site, in the form of conversations.

(3) Subjects' diversity: This is defined through (first) the combination of the role(s) through which subjects relate to the park and (second) each intra-action (in which subject and object mutually emerge), and properties (i.e. framing, references). In the level of roles, subjects' diversity mostly corresponds to a diversity of stakeholders and diversity of types of citizens. Precisely, in some of the 31 descriptions, the subjects' roles with the park were many. This happened when a subject referred to the park within the same description through different roles (information available in the table). For example, a park worker discussed the park from three points of view: from their role as a worker, from their visits to the park during their free time and from their childhood memories. A similar expansion of roles occurred in the case of a conversation or an interview. Based on the collected information, the subjects' roles in this test 
were the following: citizens, residents living nearby the park, activists claiming different things for the park, entrepreneurs, real estate agents, architects, urban designers and planners involved in interventions or discussions about the Pedion Areos Park, subjects related to the state, poets and writers, people working for facilities in the park, homeless people, drug users, and dogs. ${ }^{21}$ Furthermore, subjects' differences depended on their diverse points of view, as these were determined by things such as ideologies and intentions. A special feature of this test was the existence of references among subjects (i.e. a citizen to a state agent): This belongs to the parametrization, since such social relationships contribute to the park's form. These intersubjective relations, as embedded in the gathered descriptions, may advise for combinations of selections. For example, an activist considers that the park has problems, because it is abandoned by the state. The methodology enables the exploration of the park through the combination of the properties attributed to it by all subjects appearing as engaged in it.

(4) Time: The gathered descriptions cover the timespan of 1845 to 2018. The integration of time in the second case study enhanced the consideration of problems of the park diachronically, or how the attributed properties (i.e. by a certain type of subject) changed throughout time.

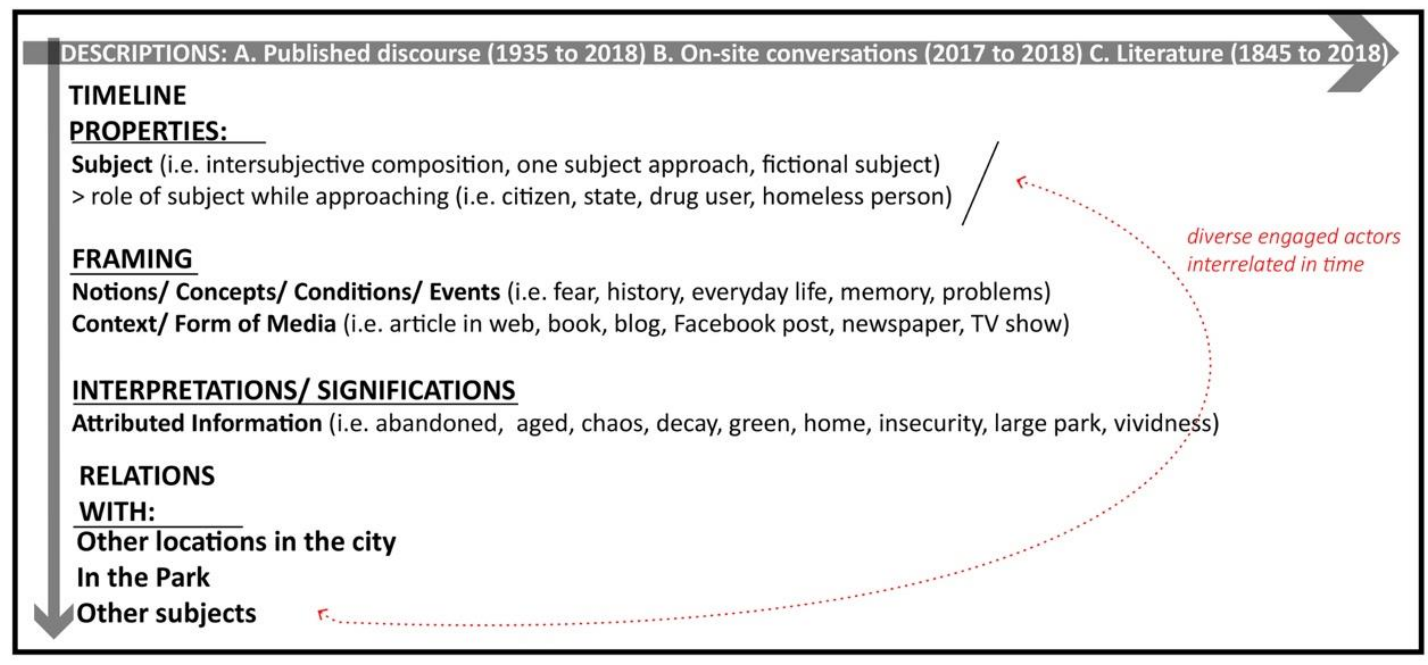

FIGURE 20. The main parameters for the organization of the 31 mappings/descriptions of Pedion Areos Park into a table (visualization: Stamatopoulou, 2018).

(5) Territorial scale: The second test was concentrated on a specific location in Athens (a park), compared to the broader area in the first case. The combinatory with the view not only revealed the different scales of complexity and relationalities, it most importantly challenged the methodology of how it deals with the transitions between them, in dialogue with Chora's (Bunschoten, 2003) and SOD's (Sevaldson, 2009) relevant objectives on multi- and crossscalarity. References to other locations of the city belong to parametrization and are noted to the table.

The parametrization scenario is similar to this of the first case, and it adds time. It prioritizes the conceptual and semiotic parameters and organizes categories of properties as shown in Figure 19: Framing corresponds to whatever frames the description as intra-action, while interpretations/ significations focuses on properties and capacities in the form of visions. Relationships with other subjects and other locations constitute additional categories. The former enables more experiments with inter-subjectivity. 


\section{Testing through the Interactive Open Map \\ Athens Center public space case study}

One option in the IOM is the selection of properties in the table, in order to activate references on the map. In Figure 21, the property "open editable file" (highlighted on the table's vertical axis) is selected. As a result, the (three) mappings having this property are highlighted on the horizontal axis, and their references to the city are activated on the map, as noted in the form of black dots. This way, the spatial expression of one or more properties can be revealed. In each of these three mappings, the referred locations can be considered connected, depending on how each subject relates them. These relations are independent of IOM, because they exist in the original mappings.

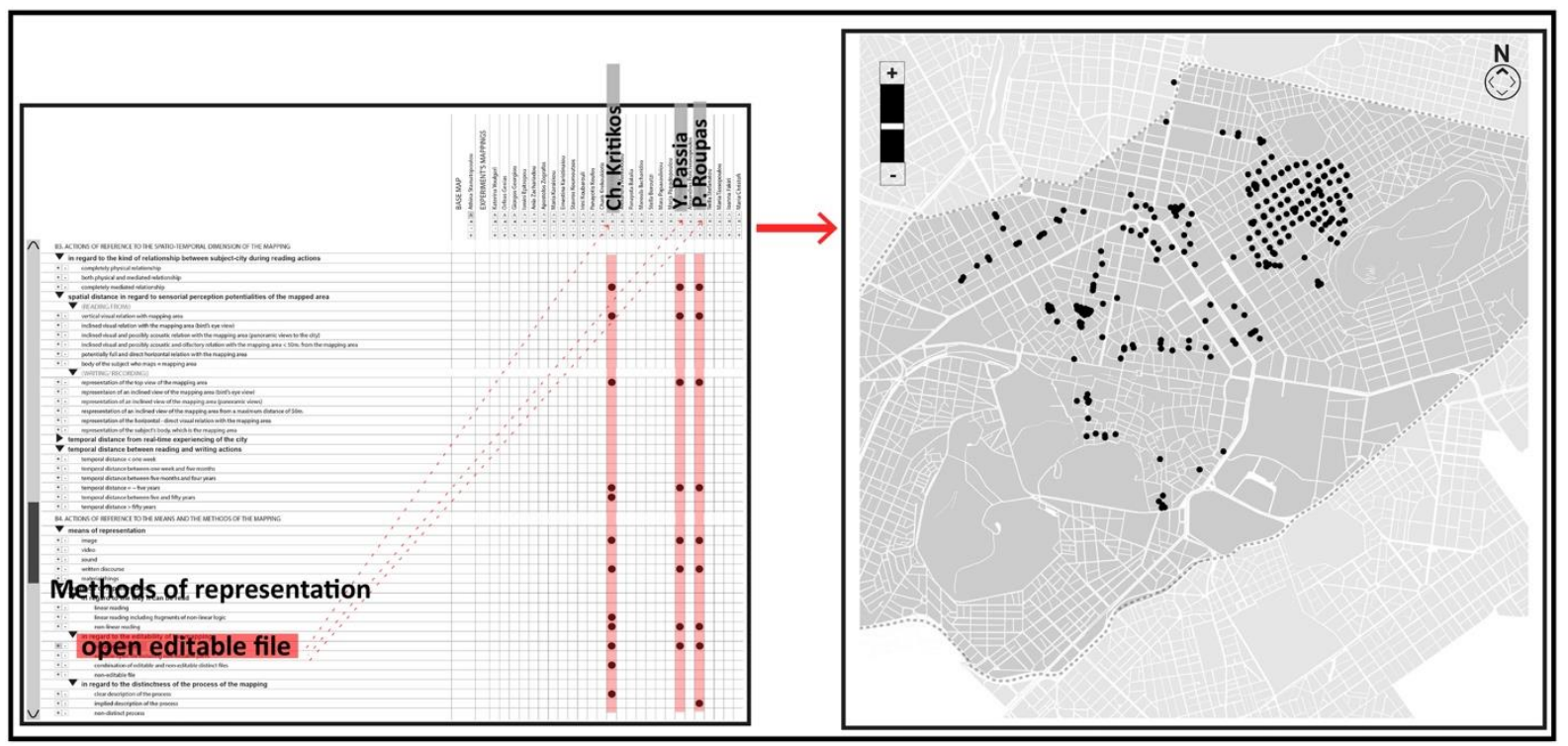

FIGURE 21. Sample snapshot of the selection of a property ("open editable file" highlighted in red) in the vertical axis of the table: It indicates the (three) mappings including this property on the horizontal one. It activates their matched properties (table) and their references on the map. (visualization: Stamatopoulou, 2020)

The IOM superimposes these mappings (Figure 21). In this manner, it goes beyond their independent realities and may be used to explore the emergent potentialities of their synergies. The generativity of such operations can be evaluated by setting degrees of generativity with regard to the superimpositions' intensity. The latter can be counted in each mapping under the criteria of: (a) references' densities; (b) references' intensity, traced from a mapping's content; and (c) participation of diverse mappings in the superimposition. Figure 22 shows this reflection: Different areas indicating different degrees of (potential) generativity are marked, taking into account criteria (a) and (c). In the darker red areas, the superimposition has a higher potential of generativity, because all three of the mappings make reference to locations in them. The diversity of these references could inform the intensity as well. Toward the lighter red, the degree of generativity decreases, since the superimposition options get limited. Considering that through the actions performed in the IOM all the references (of the three mappings) to the city are getting connected, what one sees on the map is a potential network. For instance, it can be assumed that all dots (locations on the map of Figure 21) form a network; a differentiated network from these is within each of the three mappings. Therefore, operations in the IOM not only create connections on the terrain's map, they offer value in their capacity to generate potential networks and new information (here shown in a simple way). 


\section{superimposed mappings}

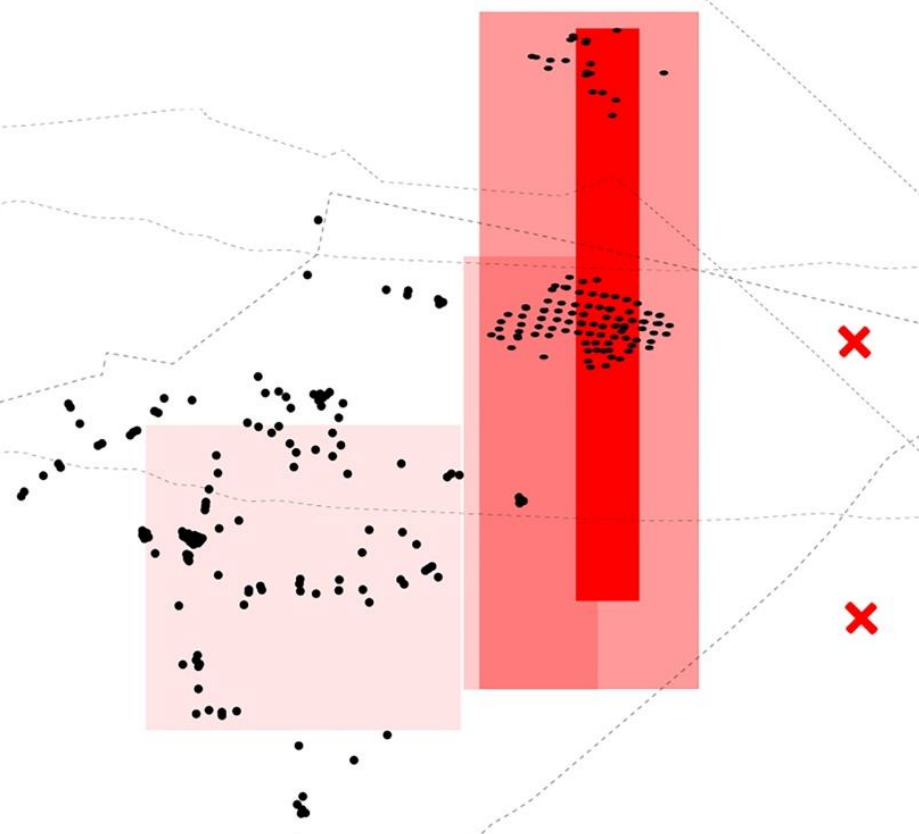

FIGURE 22. Sample of the superimposition of the three mappings activated in Figure 20. Darker red areas: higher generativity due to references' diversity. Lighter red: lower generativity. (visualization: Stamatopoulou, 2020)

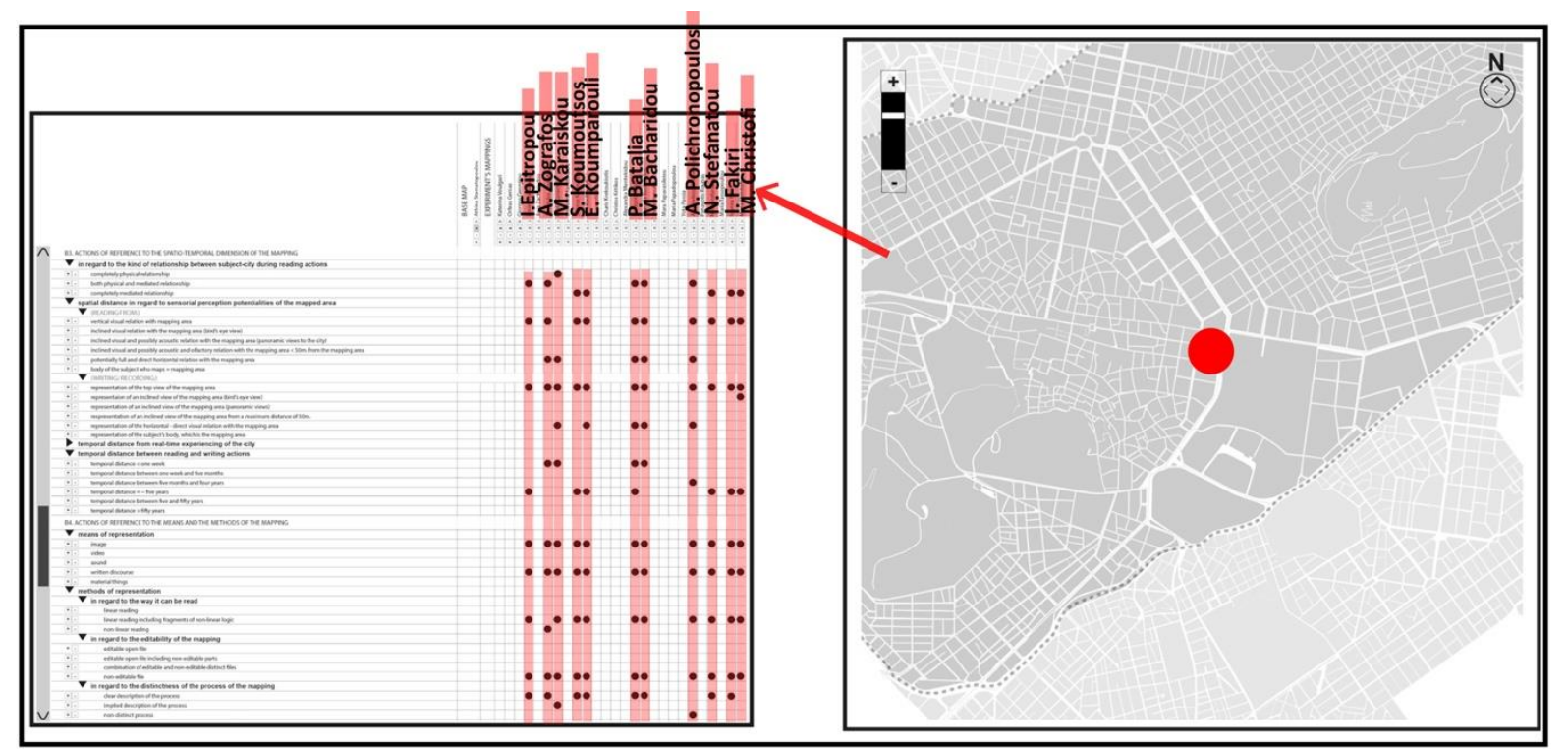

FIGURE 23. Sample snapshot of selecting a location on the map (red dot): it highlights (11) mappings including reference to it (horizontal axis and their matched properties [vertical axis]). (visualization: Stamatopoulou, 2020)

Another option in the IOM is to start by selecting a location. In Figure 23, the location Syntagma Square (red dot) is selected on the map. Consequently, the 11 mappings that include reference to Syntagma Square get highlighted in red (horizontal axis) on the table, and their matched properties (vertical axis) are shown. From this operation, it is firstly revealed that this red dot is a superimposition of several references to Syntagma Square. Accordingly, it is a superimposition of properties, not only within each 
mapping, but by the superimposition of the 11 mappings, as reflected by the highlighted columns of the table. In the IOM, each reference on the map is accompanied by the different properties attributed to it by the diverse mappings. Another remark is that Syntagma Square is not the single reference that these 11 mappings make to the city. By selecting Syntagma Square, it should be taken into account that in each mapping this location belongs to a network of more references.

Information is incorporated in locations, mainly as latent relations among data. In the IOM, this can be detected in two levels. The first concerns information, or at least data, coming from properties attributed to locations by each different mapping. Here, the IOM does not generate information, it only reveals it. In the example of Syntagma Square, such data can be extracted from the 11 mappings and the properties on the table, or the database. The second level of information has to do with the information superimposition performed in the IOM. Such information is generated by the IOM and exists only in its context.

Through the operations explored in Figures 21 and 23, one can go deeper on how locations and information are dynamically interrelated. Specifically, through the IOM it can be examined how information-here in its semiotic aspect-affects locations' relations, and vice-versa: how relations of locations unveil relations of data and potentially relations of information. For example, think of two public squares and a stroller: In metric quantitative terms, the public squares might be the same distance from the stroller. However, this distance might get distorted when it comes to how it feels, or largely when it is evaluated in qualitative terms, i.e., which one is more popular. Regarding investigation of the information, locations' interrelations may expand to the detection of patterns (i.e. how specific combinations of properties trigger specific relations of locations in repeated ways) through more systemized back-and-forth transitions between the table and the map. These transitions may deploy either for tracing information from the mappings, or as simulating superimpositions. The latter, as speculated in the next section, enforces experimentation with interventions in relations of locations by intervening with the information linking them.

\section{Toward Pedion Areos Park case study}

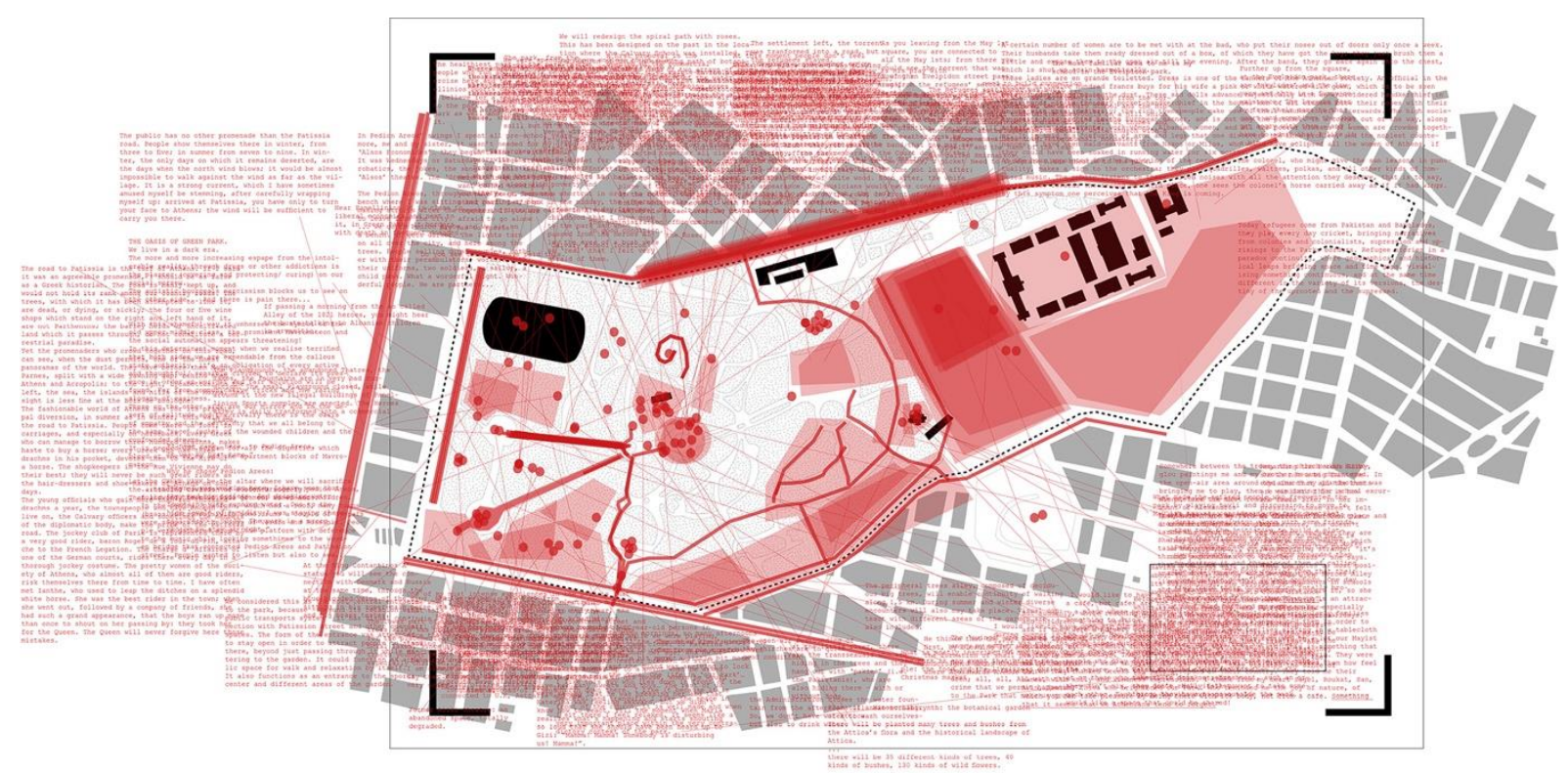

FIGURE 24. Map of all references of the 31 descriptions to Pedion Areos Park. References to the physical terrain are noted in red (dots, lines, irregular schemes) in the same logic as with the first test. Visualization techniques were improvised, based on the collected material and the territorial scale. (visualization: Stamatopoulou, 2018) 
The node of a location, like Syntagma Square, can be further analyzed. This is already implied by (a) its composition as an overlapping of references to it from the 11 mappings, and (b) its participation in networks of other references to the city. The second case study goes deeper into the relationality of each node as a zoom-in action (Figure 25). Its field is relationally composed (Figure 24) in exactly the same way as with Athens Center in the first case. More analytically, the second case is a park (that is a specific location). As shown in Figure 25, in the first case study, such a park would be noted as a dot (like Syntagma Square), since it is a specific location.

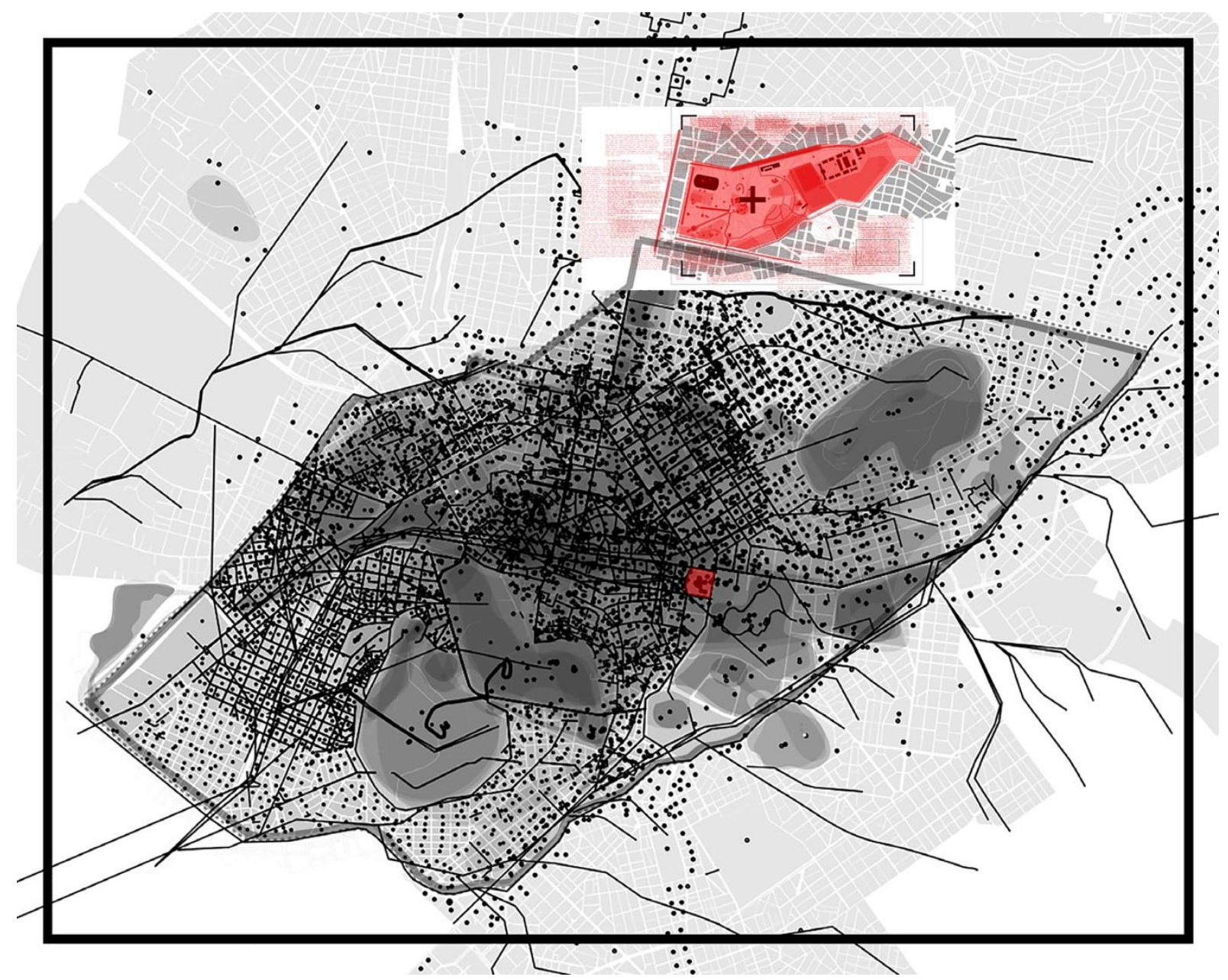

FIGURE 25. Pedion Areos Park embedded in the first testing case. Although territories are physically neighboring, the two tests have no other relationships of content here. (visualization: Stamatopoulou, 2018)

Therefore, nodes are relational, not only because they participate in networks, but because they are composed of networks themselves. This means that every node/dot on the map of Athens Center (Figure 25) not only incorporates further networks, but that all networks are nodes of larger interrelations. By zooming out, every network looks like a node. This enables thinking in terms of openendedness and multi-entry options, like rhizomes (Deleuze and Guattari, 1987; Corner, 1999). This resembles the way that Chora zooms in and out of a territory (Bunschoten, 2003). Such an emphasis on relationality is a proposal of the in-progress methodology for how to zoom in and out territories. As for framing, zoom begins by scanning the territory's relations through data, in order to check where these indicate a zoom in. Multiple zooms in and out permit the simultaneous combination of different networks and scales of complexity. This enhances a multi-scalar (co-existing scales) and intra-scalar (merging information among scales) exploration of a complex thing like the city. 


\section{SPECULATIVE PERSPECTIVES OF DESIGN LOGICS}

The operations explored in the previous section are random snapshots demonstrating, in simple ways, how this methodology may operate, such as to reveal multi-scalar, complex, physical-and-semiotic networks of information, to trigger multiple re-organizations of the dominant relations in/of the city. In this section a deeper analysis is offered on how latent capacities of the methodology could be adapted and possibly evolve, in order to discuss design processes through the terms of relationality and complexity over the broader context of system thinking.

\section{Designing by analyzing/mapping}

The methodology, by embedding the IOM, adopts operative mappings' capacities, such as to dissolve the "dichotomy between analysis and design" (Paez, 2019, p. 218). This way, design proposals are attached to analysis actions. Through diagrammatic operations, analysis in the form of mapping converges with the actions of synthesis through generative decisions (Figure 26).

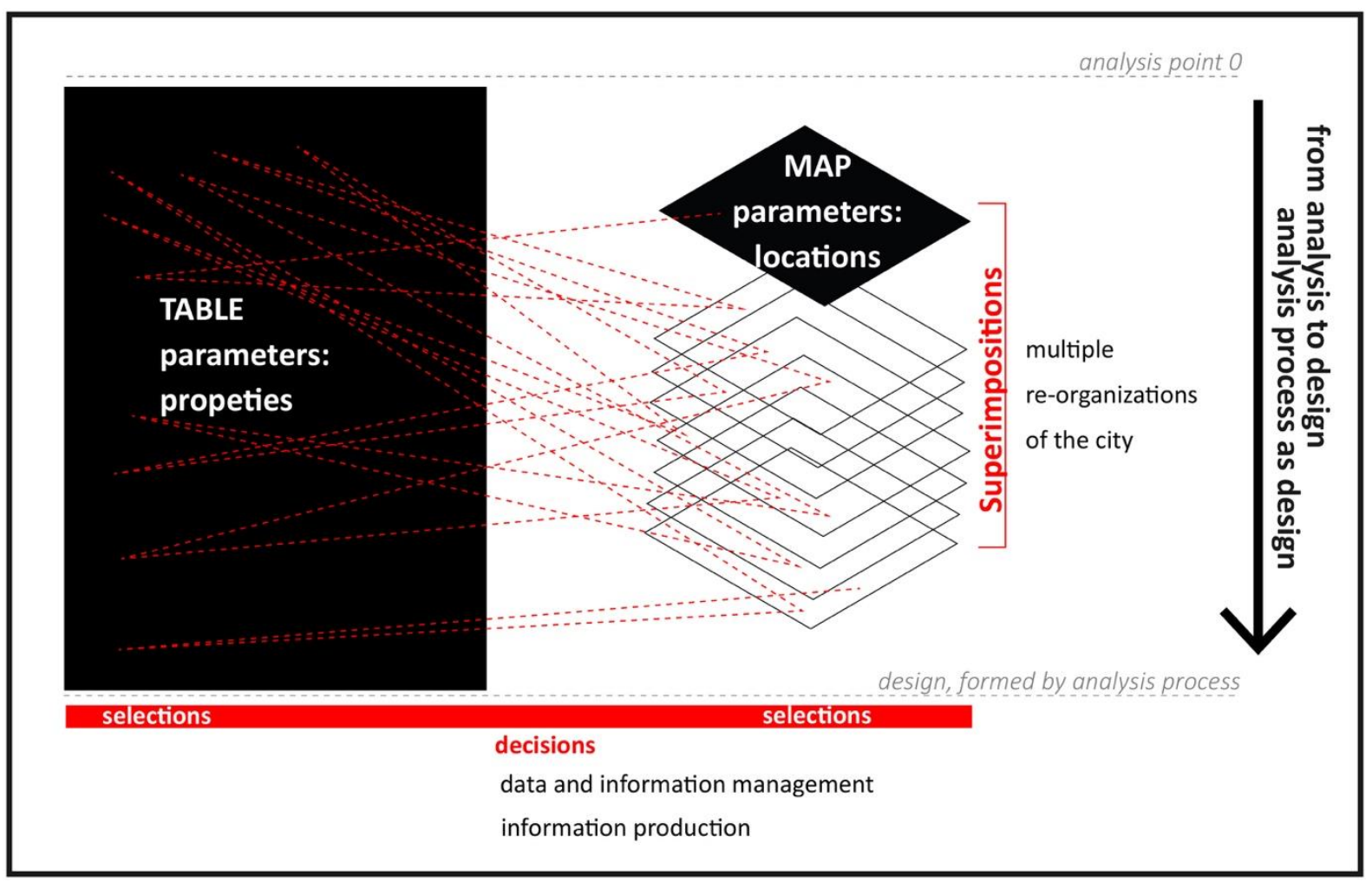

FIGURE 26. How an analysis process becomes designing, through selections (visualization: Stamatopoulou, 2020).

In the IOM, the city's analysis is articulated to the management of the organized mappings' assemblages: By making selections on the map, on the table, or in the database, complex relations that are beyond, yet attached to the physical, arise. These organizing operations recall the principle of created emergence (Jones, 2014). In Figure 23, when Syntagma Square is chosen on the map, the 11 mappings containing reference to it get highlighted on the table (horizontal axis) along with their matched properties (vertical axis). Then, in Figure 27, the other references of the 11 mappings to the city are selected to be activated on the map. As a result, the overlapping of all the 11 different networks, in which Syntagma Square is part, are unveiled. This selection not only triggers the superimposition of these networks, it uncovers the diffusion of the selected location through the systems/networks in which it is a part (Figure 28). 


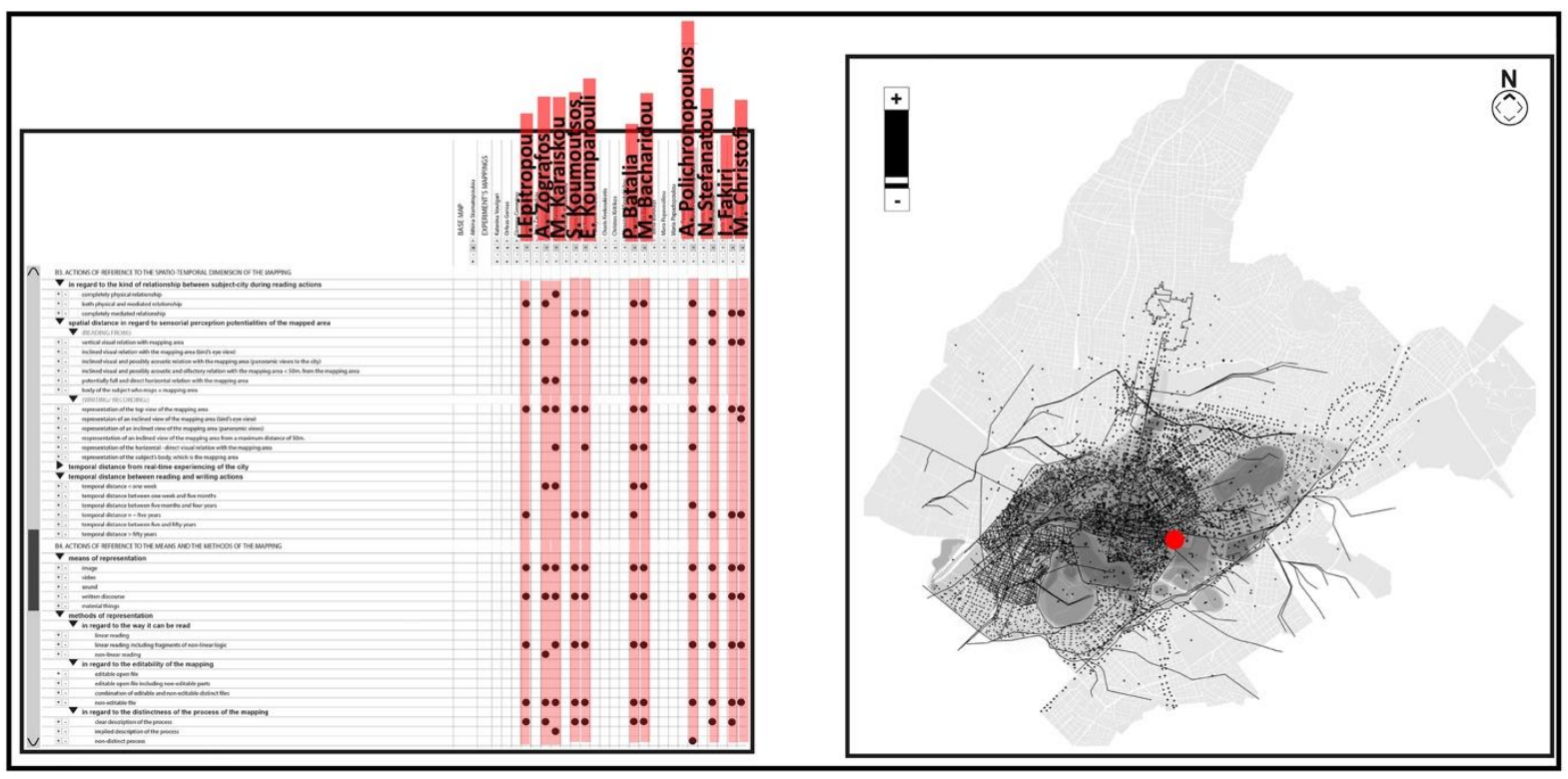

FIGURE 27. Sample snapshot of triggering a location's complexity. Selection (red dot) on the map: highlights 11 mappings (table's horizontal axis), including reference to it and the matched properties. Then, the other references (in black) of the 11 mappings to the city are shown (map). (visualization: Stamatopoulou, 2020)

Such selections are decisions producing information, not (already) existing outside the IOM's context. For example, the black network in Figure 26 constitutes (new) information that emerged within the IOM system. Figure 27 is a moment of the process demonstrated in Figure 26. The selections' generativity is intensified by the non-linearity of the operations' sequences (Figure 26: red dashed lines), which enhance experimentation and simulations. Operations among the IOM's components are non-linear. For instance, in Figure 26, after activating the networks related to Syntagma Square, getting back to the table to activate or deactivate further information is possible. Such selections or deselections concern parameters for filtering information on the table-map system (Figure 26). Thus, back-and-forth actions are potentially unlimited, based on the available options for selections (Figure 15). This mainly concerns non-testing applications: In these, selections are expected to be more complicated, organizing and/or ordering decisions, following concrete scenarios articulated to their particular context. Relations and interdependencies among parameters (i.e. in Figure 16) could provide directions for selections.

Through decisions supported by the selections in the IOM, the city can be de-assembled and re-assembled, namely re-organized in multiple ways. The defined parameters and their potential interrelations are the fundamental components for generative selections in general, while the parametrization hypothesis suggests trajectories for possible back-and-forth movements. In this manner, analysis - formed as a sequence of superimpositions that variously produce information - aims at designing, rather than at analysis. Both verbs take the form of managing and deciding within the same system-interface (i.e. Figure 26 and 27: IOM scheme). Such a series of decisions may design further systems through system ordering and generative emergence (Jones, 2014). They may also evolve into detailed proposals' composition, as with logic in which analysis/mapping(s) of layering and overlapping existing conditions informs design (i.e. Tschumi's Parc de La Villette).

\section{Designing by defining frame(s): Emergent framings}

The aforementioned selections are also framing actions. They set the frame, within which further operations of analysis and design will take place. In the logic promoted here, this frame is neither taken for granted nor arbitrarily imposed; it is also not ignored. It arises organically through the analytical process (Figure 26), namely through selections, and particularly superimposition. The set of operations that was unveiled in the networks in Figure 27 constitutes such a frame definition. This example offers the occasion to clarify that framing in the IOM is not limited to the notation of a territorial borderline. First, there is no concrete borderline. Geographical boundaries are investigated through overlapping 
areas (Figure 28: right map in red), as these emerge from superimposition operations among selected networks (Figure 28: left map in black). By this, the in-progress methodology offers an alternative to the logic of pre-defined boundaries in the so-called top-down ways (i.e. in architectural-urban design competitions), further emphasizing their complexity.

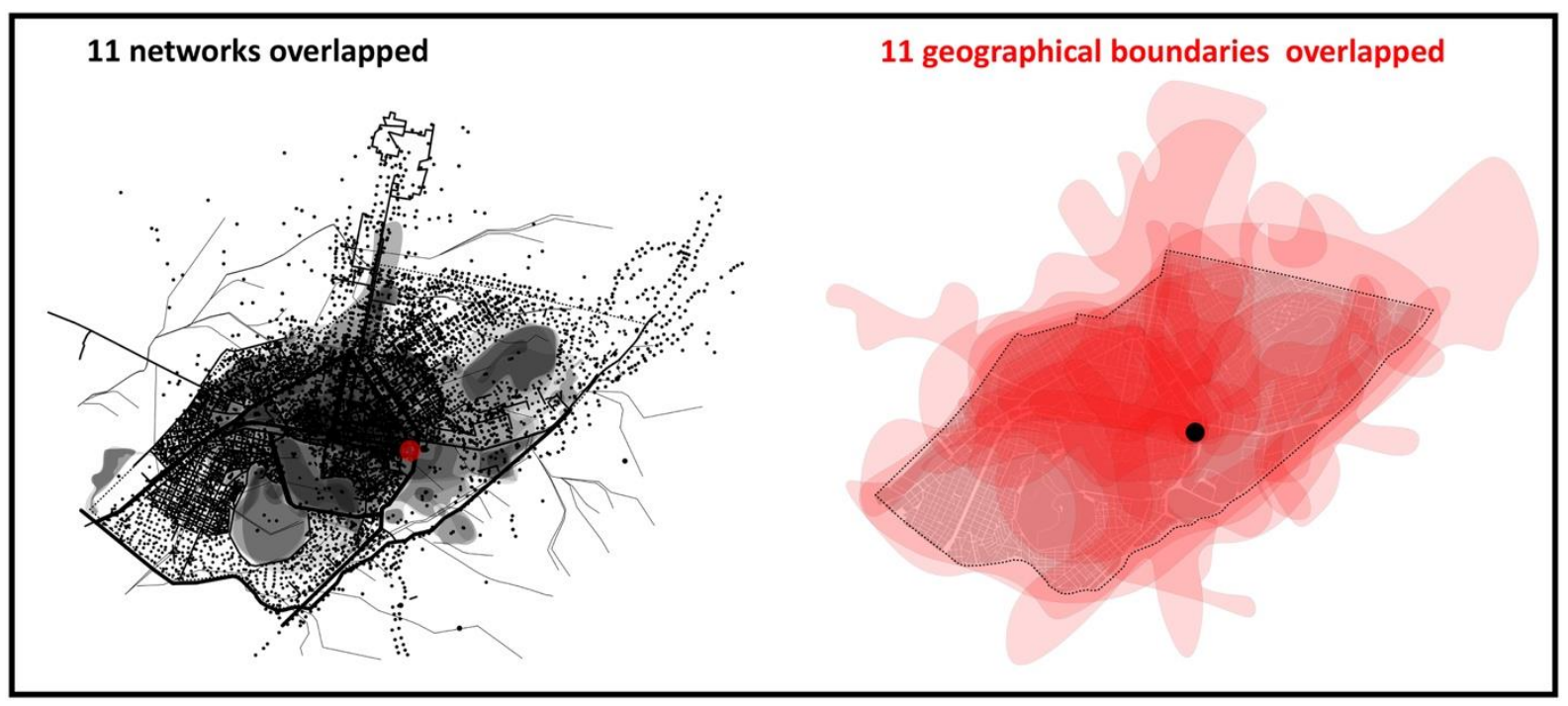

FIGURE 28. Left map: overlapping of 11 networks by 11 mappings including reference to Syntagma Square (red dot). Right map: translation of the 11 networks into 11 boundaries of areas that are overlapped (in red). (visualization: Stamatopoulou, 2020)

Second, through the map-table-database interrelations, the framed territory is attached to diverse information (Figure 29: upper part). Such information sets the terms, the briefing program for an analysis, and/or design process along with the geographical constraints. The terms can be elicited from the tables' parameters (i.e. concepts, attributed significations) and from operations revealing relations with other locations on the map. The selection of such terms may contribute to appreciating complexity, purpose finding, and requisite variety in the context of systemic design principles (Jones, 2014). Thus, defining frames' operation detects and proposes locations, areas, networks, or other systems for further interventions. At the same time, design involves the description of both the information- and territorialwise framework through which new relations can emerge. The superimposed mappings of Figure 27 attached to their relations with their properties on the table become an emergent whole (DeLanda, 2016, p. 10). Based on this, it is argued that an emergent framing feeds new relations. In the same spirit, fields for deeper research can be indicated, like in the Zoom operation of the ZIP analysis method (Sevaldson, 2017). In the IOM, an area for zoom-in is attached to the table's properties, while the zoom process triggers more details to unveil. This way, the properties from the zoomed-out scale remain capable of informing the definition of problems and potentials, innovations-interventions, and ideas in the zoom-in mode. The same can be applied in transitions from zoom-in to zoom-out, cultivating the multi-scalarity. Moreover, this logic strengthens the relational territoriality in which analysis and design is supposed to assemble physical and non-physical relations as a system (Figure 29: upper part). 


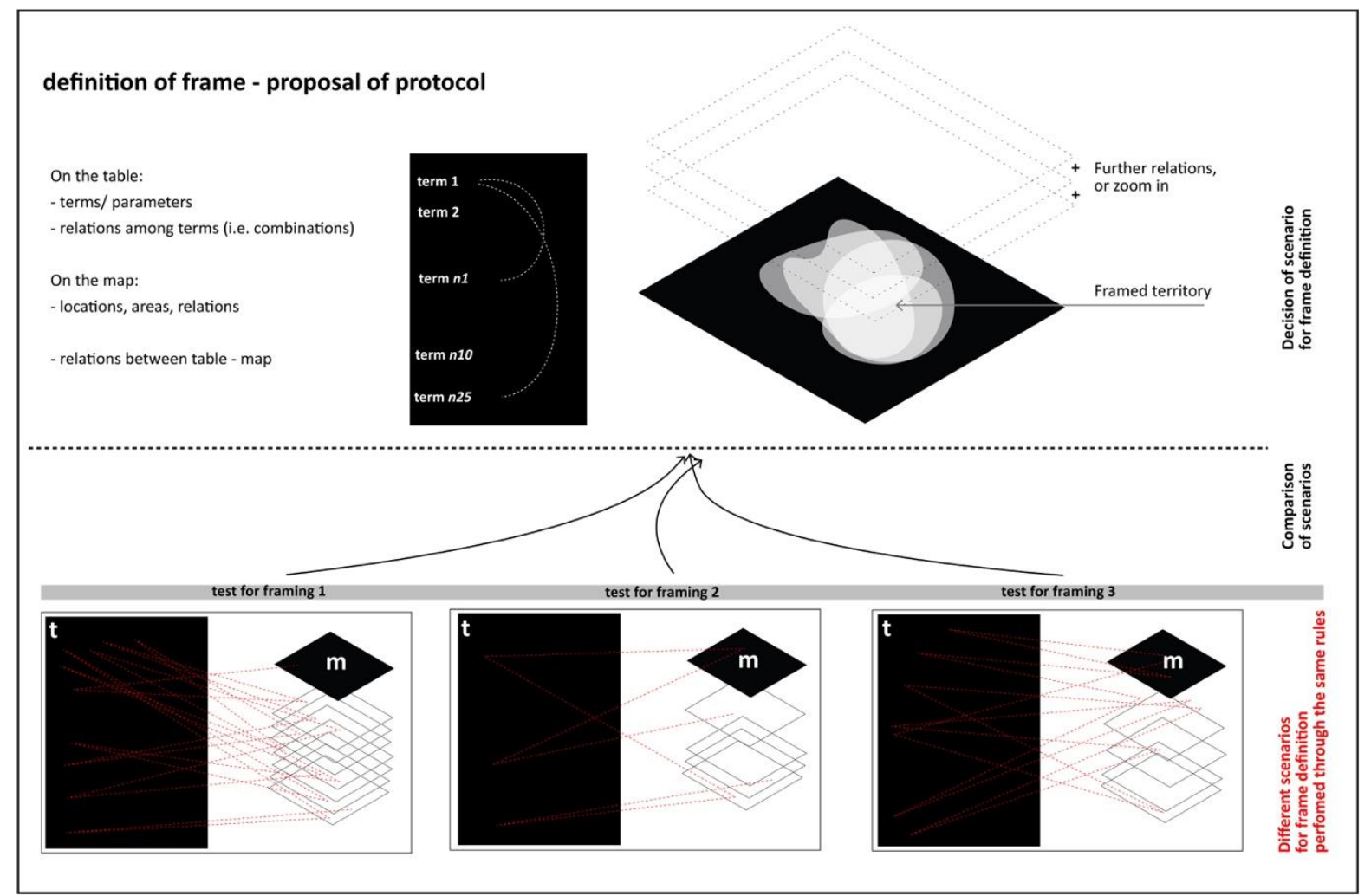

FIGURE 29. Up: scheme of the components for the definition of a frame. Below: Visualization of the speculation on how the definition of a frame can result through diverse scenarios. (visualization: Stamatopoulou, 2020)

Defining the frame for further design actions may take the form of designing protocols (Paez, 2019), recalling the protocols-oriented methodology of Urban Gallery (Bunschoten, 2003). As explored in the lower part of Figure 28, the definition of frames, inspired by the Scenario Games (Bunschoten, 2007) can be the outcome of different scenario test comparisons.

\section{Design the design program: briefing making}

Back-and-forth movements in the IOM mediate multiple zooms into and out of territorial relations, in terms of detail(s) and scale(s). The selections that were revealed and superimposed in the 11 networks of Syntagma Square (Figure 27) may take the form of a further zoom-in, to proceed to a more concrete briefing-oriented design proposal. A sample scenario here is a proposal for Syntagma Square with regard to the combination of the concepts of the commons and of the Otherness. The complexity of the 11 superimposed networks can be managed by being filtered and abstracted through (de)selections on the table: Among the 11 mappings, 3 of them refer to it through the two selected concepts. Information in the IOM is accordingly abstracted: In Figure 29, both the table and the map show only the 3 mappings and their references to the city. The references are painted in three different colors, in order to make the different source-networks distinct. This emphasizes the relationality of the node. The same location is integrated in different networks in two dimensions: in each mapping and in their superimposition in the IOM. It additionally enables observations of the spatial manifestation of each mapping in the geographical terrain, but most importantly it reveals the spatial relations of the three mappings. This way, additional areas of encounters among the different network-mappings can be traced. Depending on the concepts and the meanings they carry, these encounters may have complementary or conflicting relations affecting the physical ones. Such kinds of information, revealed through further operations in the IOM, are very important, since relations are not neutral. 


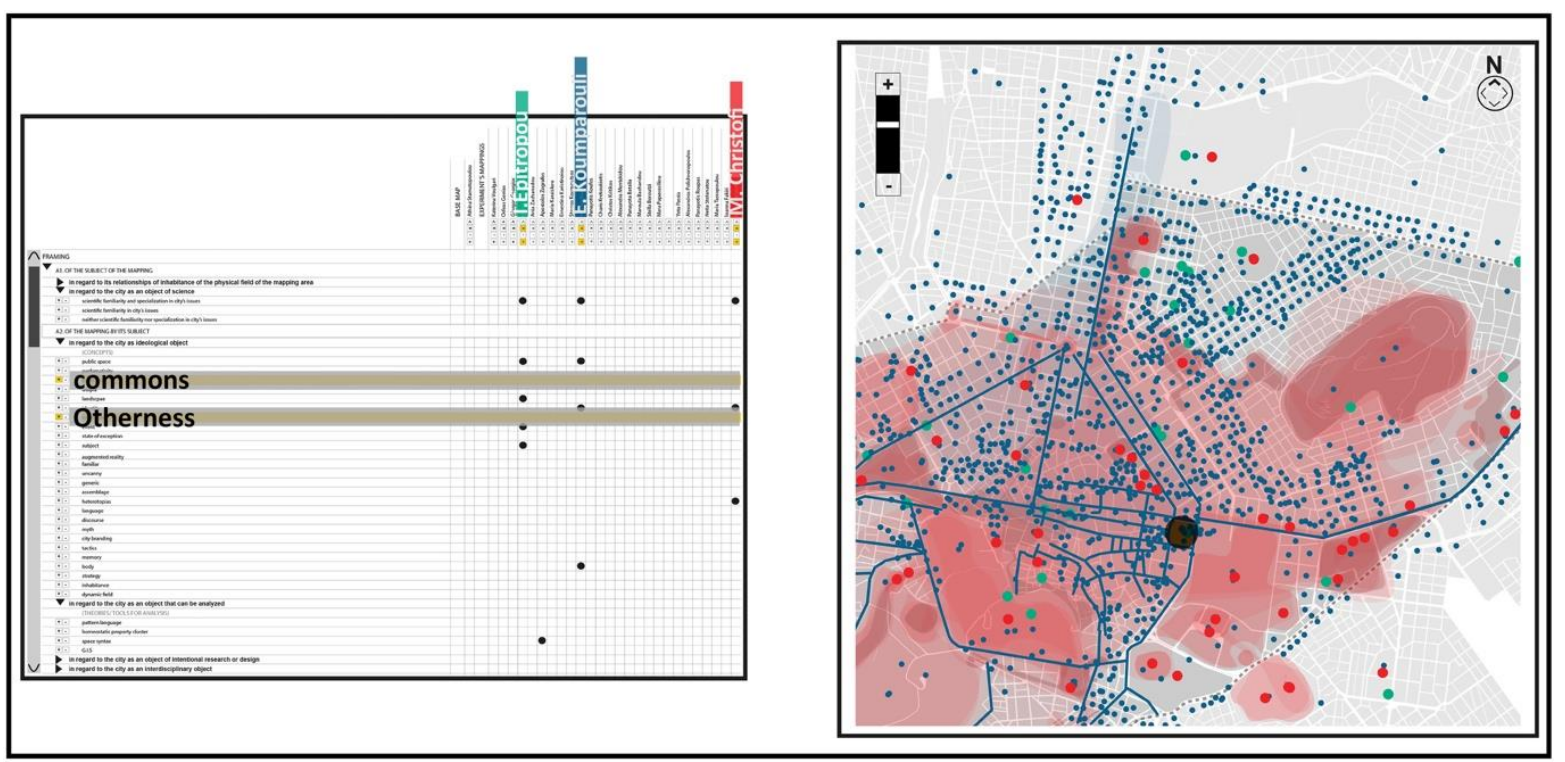

FIGURE 30. Sample snapshot of making abstractions: The networks of Syntagma Square (Figure 27) are abstracted with regard to the 3 mappings approaching it through the parameters of the concept of the commons and of Otherness (marked in vertical axis of the table). The references related to the three mappings are noted in three different colors (green, blue, red). (visualization: Stamatopoulou, 2020)

In this perspective, the design is the decision of the briefing, which heretofore, includes a specific node/location and the revealed territorial networks of Figure 30 as well as the combination of the concepts of the commons and of the Otherness. These compose a latent system of information, generated by the abstractions made. The briefing can become more concrete through additional decisions in two directions.

D1: First, the three mappings refer to the concepts of the commons and of the Otherness in diverse ways, which theoretically might turn out to be contradictive. Contractiveness here is viewed as variety, which is significant in the broader spirit of appreciating complexity (Jones, 2014). For this reason, the selected concepts for the briefing can be enriched by the special manifestations of the concepts in each mapping's original form, as this can be elicited from the database. Second, this diversity also depends on how the two concepts are associated in each mapping. This expands to the relations of the two concepts with the other properties, and in particular, with those of semiotic ${ }^{22}$ value, as they are detectable from matches on the table. In this direction, these operations serve the enrichment of the variations of the two concepts. In parallel, they cultivate the second dimension.

D2: The combination of the concept of the commons and that of the Otherness is the conceptual core for the speculated design process. However, other properties (i.e. concepts, meanings) drawn from the mappings can conceptually enrich this core. For instance, the mapping of the blue network on the map of Figure 30 includes the notion of the body, linked to performance and theater. Such concepts can be added as secondary to the intended briefing. Moreover, the territorial relations, or operations triggering the emergent framings discussed before, may define the boundaries of the area to be designed, or the locations with which the proposal should be connected, physically and/or conceptually. Specifically, emphasis is given to the territories' boundaries.

To summarize, the design as a briefing is composed (Figure 31) of (1) primary concepts, (2) requested properties (including secondary concepts), (3) a defined intervention area (through emergent framing), and (4) areas to take into account, apart from the initial, targeted location. Such briefings' logic may contribute to the formation of urban design/architectural competitions, in which design may still evolve in different forms. Briefings can be further elaborated as protocols, like the emergent frames. 


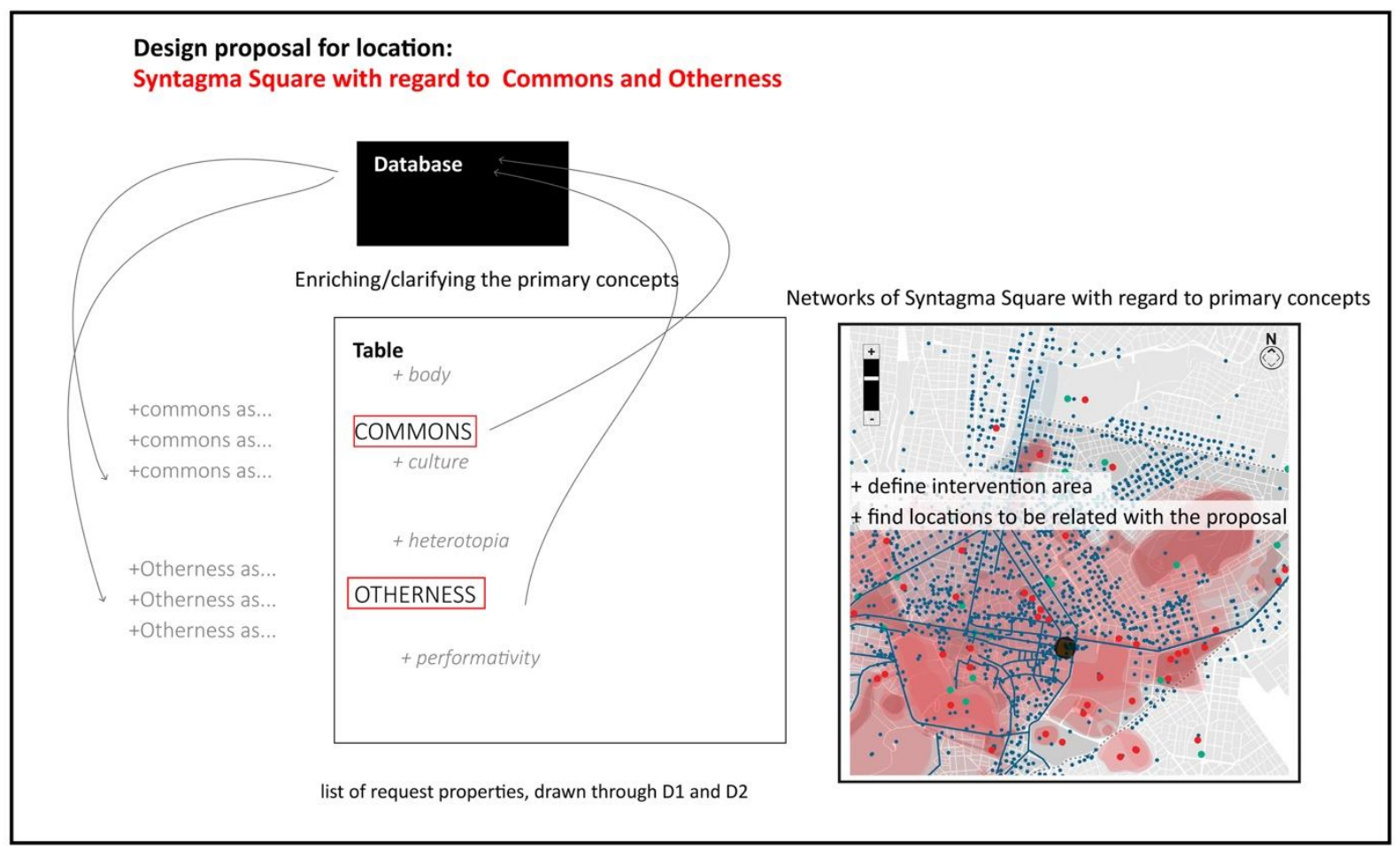

FIGURE 31. Visualization of operations for further definition of the briefing (by Stamatopoulou, 2020).

\section{Design as an intervention strategies in/through interdependencies}

Selections in the IOM reveal the spatial manifestation of properties (from table to map) and the multiplicity of properties that a location assembles (from map to table). Properties are not limited to data extracted from the table: The relations of a location with other ones are properties. These are nonphysical relations affecting physical proximities, due to their interdependence. Based on this, a logic of strategy-oriented design is discussed. In the speculated logic, any location is approached, through the lens of other related-to-it-locations. Assuming that a designed intervention is capable of affecting other nodes or relations, one can intervene to a location without doing something directly to it. An intervention to a location can be achieved through interventions to things with which it is related, or to the whole system, in which it is part, like Easterling's (2014, p. 39) active form. Therefore, the speculated kind of strategy gives emphasis to decisions' effects as well as the processes of the interactions that are estimated to cause the intended outcome, which recalls ideas from the idealization principle (Jones, 2014).

Such strategy options can be explored through the example of Syntagma Square. The Syntagma area gathers the most references in the 26 mappings, which can be translated in terms of density. This density, already revealed in Figures 27 and 28, results from the system of 11 overlapped networks (Figure 32). The density is primarily associated with the diversity of the 11 mappings, in terms of 11 diverse contributions to the information of this relational node. Figure 32 makes this clearer, while it adds a point: The node in each mapping has a different role and is related to other referred locations in logic that can be found in the table. Therefore, this density is linked to Syntagma Square's diffusion (Figure 28). Yet, these observations are not sufficient for describing the density toward design operations. Respective selections in the IOM's table provide deeper access to the parameters and the reasons, the details of such densities. This way, density can be enriched and evaluated through its qualitative properties, taking advantage of more bottom-up elicited information. 


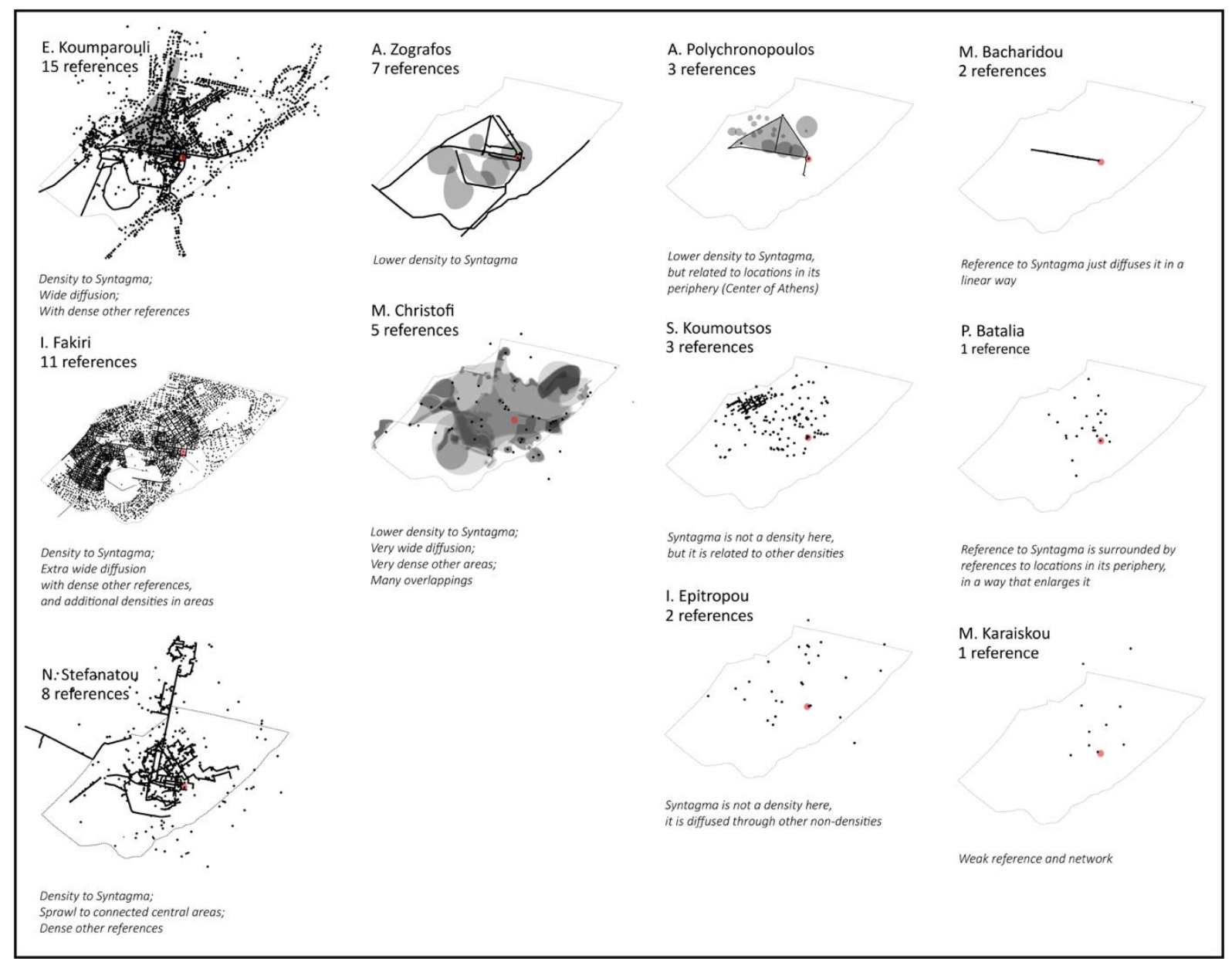

FIGURE 32. The $11 \mathrm{maps} / \mathrm{systems} \mathrm{containing} \mathrm{reference} \mathrm{to} \mathrm{Syntagma} \mathrm{Square} \mathrm{(red} \mathrm{node).} \mathrm{(visualization:} \mathrm{Stamatopoulou,} \mathrm{2020)}$

Given the non-statistical character of all the mentioned sample operations, and considering such densities as having forces (extracted and evaluated through further research), direction is given to the following strategy proposals, which can take the form (Figure 33):

(1) Of spreading and connecting the detected density to other areas. This can be achieved by cultivating densities (Figure 33a: noted in red) that have relations of attraction with Syntagma Square. A strategy to proceed to this would be focused on the dynamic interrelations of these densities, in order to examine how the system of densities balances toward the desirable condition.

(2) Of turning focus only to locations related to it. Here, Syntagma Square's density only suggests other areas for intervention. Design is focused independently on the noted (in solid red) areas in Figure 33b.

(3) Of loosening the density by intervening to its connections (red arrows in Figure 33c). In this scenario, Syntagma Square is assumed to be too dense, so interventions aim, for example, at making other locations more attractive for densities. This direction has a direction strategy similar to (a), but with a different vision for Syntagma Square.

(4) Of experimenting with the relations among overlapping densities (Figure 33d: red area). This is a strategy for an intervention to a broader area, through the interplay of networks. 


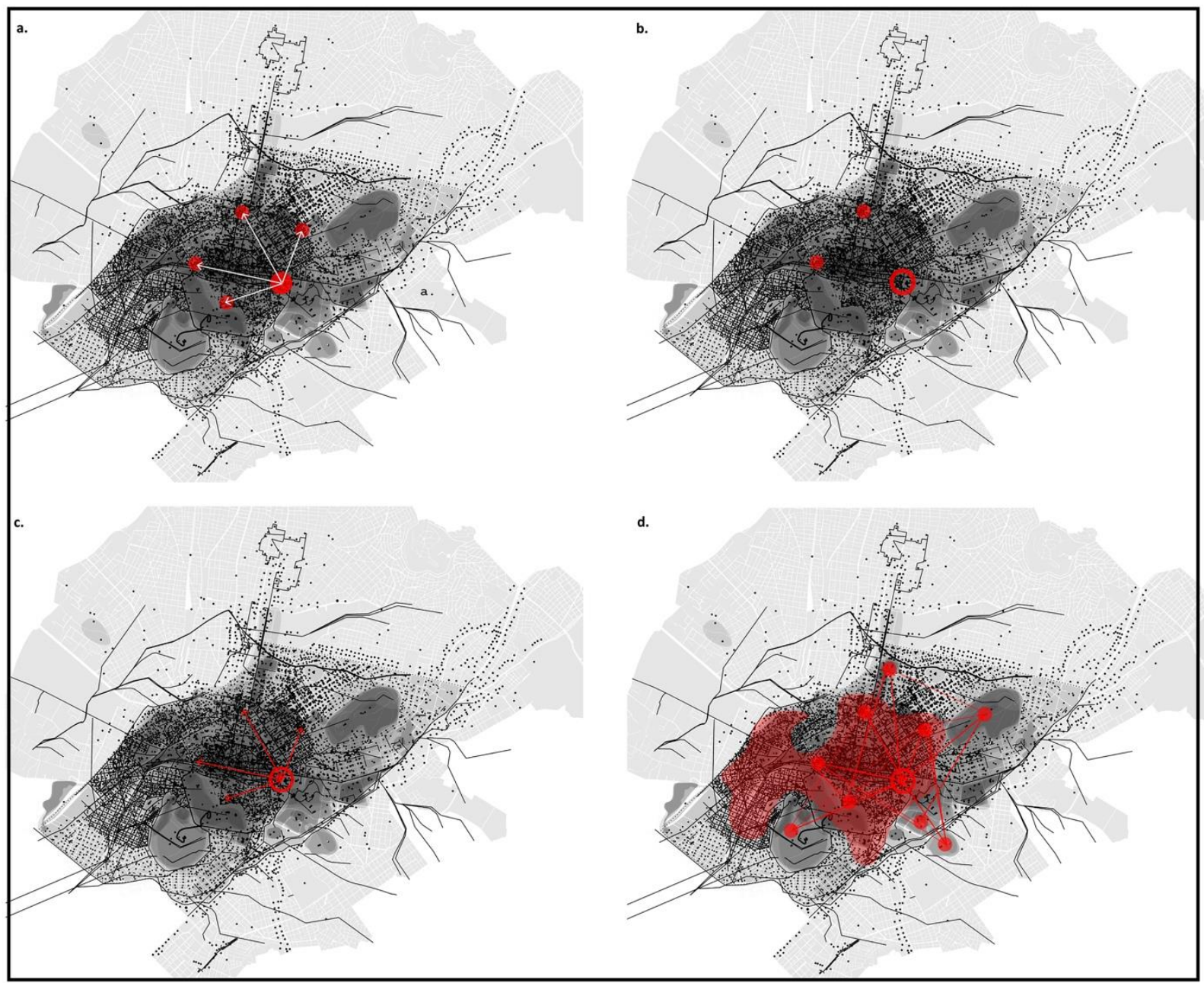

FIGURE 33. Conceptual visualizations for the potential directions of strategies. (visualization: Stamatopoulou, 2020)

Each one of these four hypothetical directions may evolve into totally different intentions, or even logics, of strategies to manage the relational density of a location in holistic ways. Experimentation with multiple alternative scenarios of idealization combined with the principles of feedback, continuous adaptation and self-organization (Jones, 2014) would benefit the application of any of such strategies. In this logic, design operations concern the whole system-environment relations, taking into account its expansion, spatial behavior, and content over time. Furthermore, all directions and the speculated logic explore not only interventions in relational-territorial interdependencies, but also interventions through them. Hence, interdependencies function as tools for interventions.

\section{Design by intervening with territorial forces}

Strategies for and through interdependencies place emphasis on the forces of non-neutral relations, as these are determined by information. This is explored through the case of Pedion Areos Park. By selecting the property "problems" in the table (Figure 34), its variations are revealed. These variations might be contradictory as a matter of interpretation, yet they depend on whether and how a condition is recognized as problem. Such complex problems are wicked problems (Rittel and Webber, 1984). These variations of problems show that their spatial manifestations (in red) on the map (Figure 35) are diverse types networks in terms of forces: Some references to locations imply conflicting relations, while others trigger attractions. 


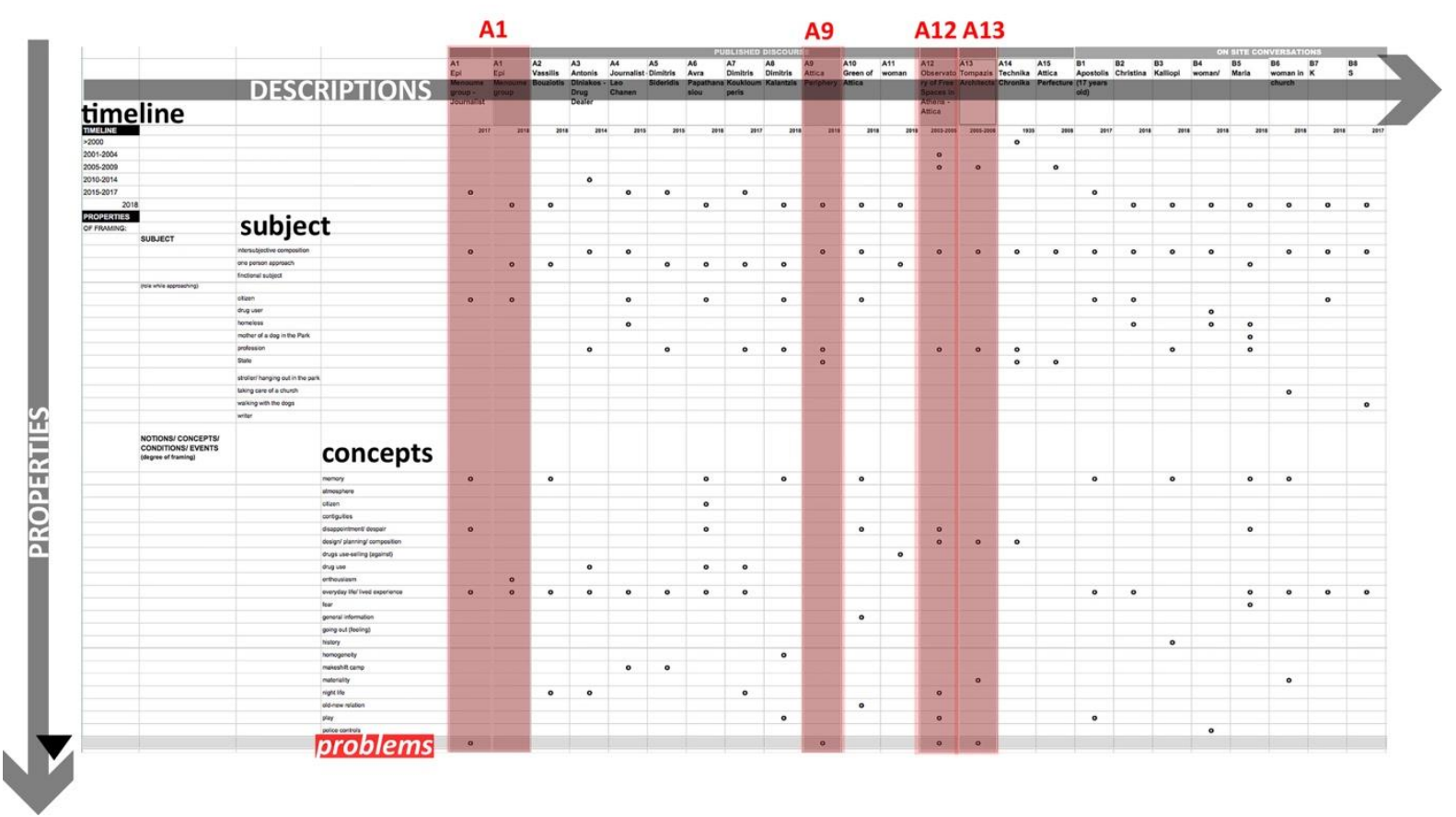

FIGURE 34. Sample snapshot of selecting the property "problems" (vertical axis; part of list is shown): descriptions (A1, A9, $\mathrm{A} 12$, and $\mathrm{A} 13$ in horizontal axis) having this property are highlighted in red. (visualization: Stamatopoulou, 2018)

Specifically, conflicting relations are revealed through information, attributing negative properties to the park. These, translated as dashed lines on maps, make the physical distances feel larger, functioning as immaterial obstacles or gaps. Relations of complementarity and consistency (solid bold lines on maps) bring locations closer than their physical proximities.

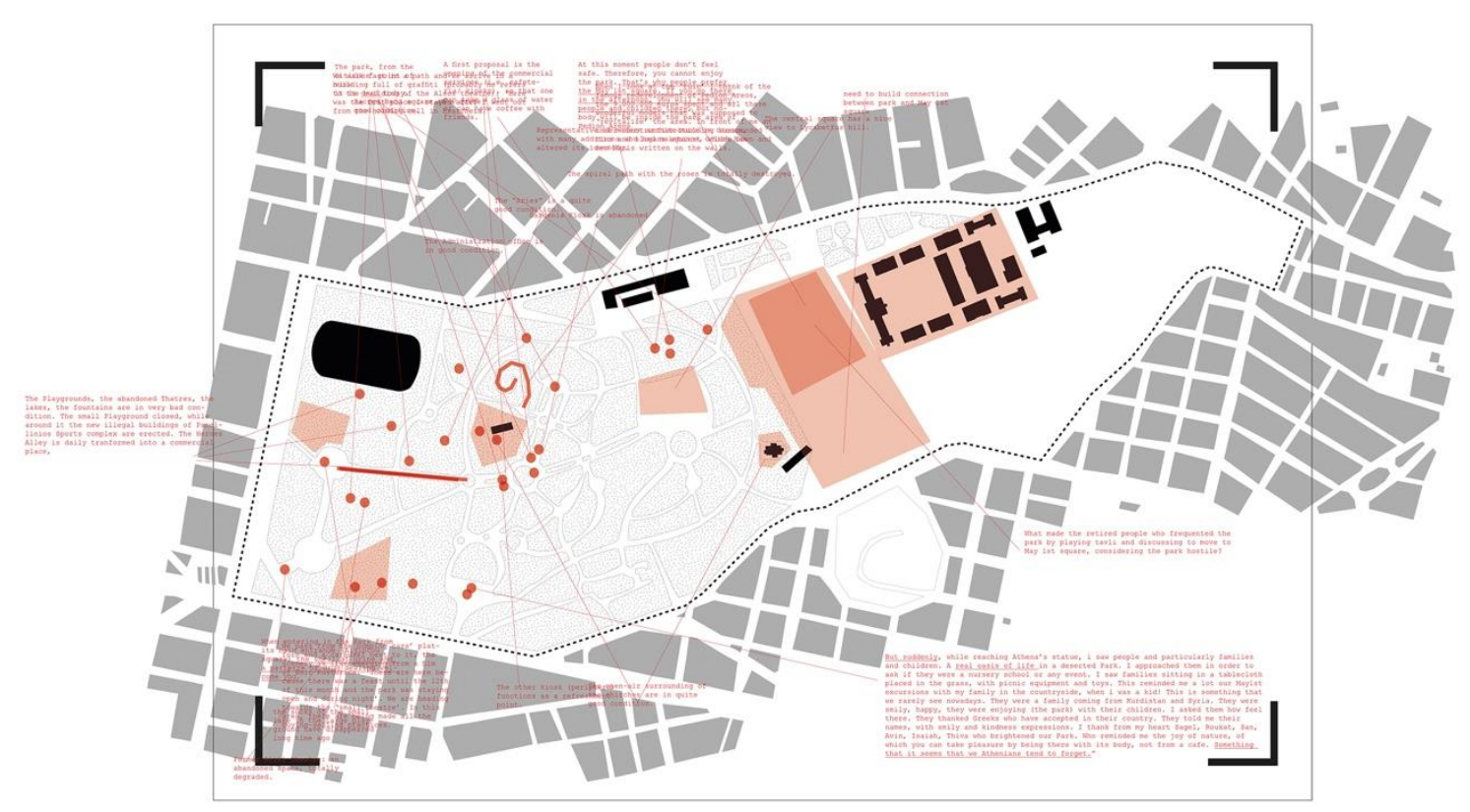

FIGURE 35. References of 4 mappings (Figure 34: highlighted on table) approaching Pedion Areos Park through "problems". (visualization: Stamatopoulou, 2018). 
Figures 36 through 39 show the maps of each of the 4 mappings in chronological order, distinguishing their references in 4 different colors. On the left side of each map, the other properties attributed to the park are noted; these are significations, ${ }^{23}$ envisioned potentials attributed to the park, and references to other subjects. For instance, the A1 description (Figure 36) refers to the subject of A9 (Figure 39), as they both consider abandonment as a problem of the park. Despite their converging content, their contexts are in conflict. A strategy taking this issue into account is expected to approach abandonment as a problem through appreciated complexity (Jones, 2014), and thus, through further operations among table-map-database, including zooms in and out. On the cited maps, all the references to the physical terrain of the park have been translated in positive and negative, according to the properties as attributed by their subjects. Connecting all the negatives and all the positives reveals fields of repulsion and fields of attraction, respectively. Similar to the four directions of strategies discussed previously, the design here could take the form of interventions in the systems of forces.

A12
Observatory of Free Spaces in Ath
2003 to 2005
abandoned stray dogs
bad lighting
bad maintenance
communal space
dangers
dark
decay
decline
dirtiness
entertainment
fenced-off areas
inaccessible at night
illegal buildings against green
insecurity
lost publicness/ commonness
monumental park
neglected
noise pollution by temporary markets
polluted
to relax
to think
to walk
<>
against:
the State,
Redevelopment Plans,
enterpreuners

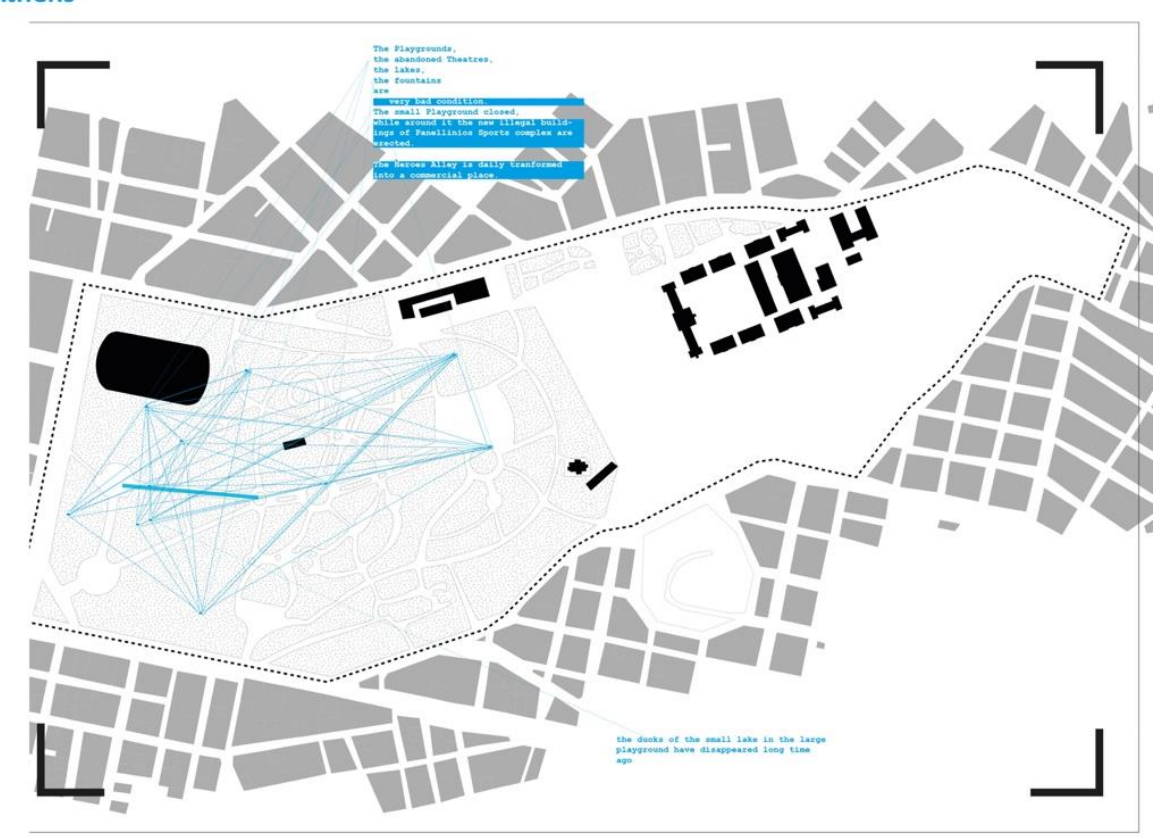

FIGURE 36. Extracted references of the description A12. Please zoom in for (secondary) details on the map (visualization: Stamatopoulou, 2018). 


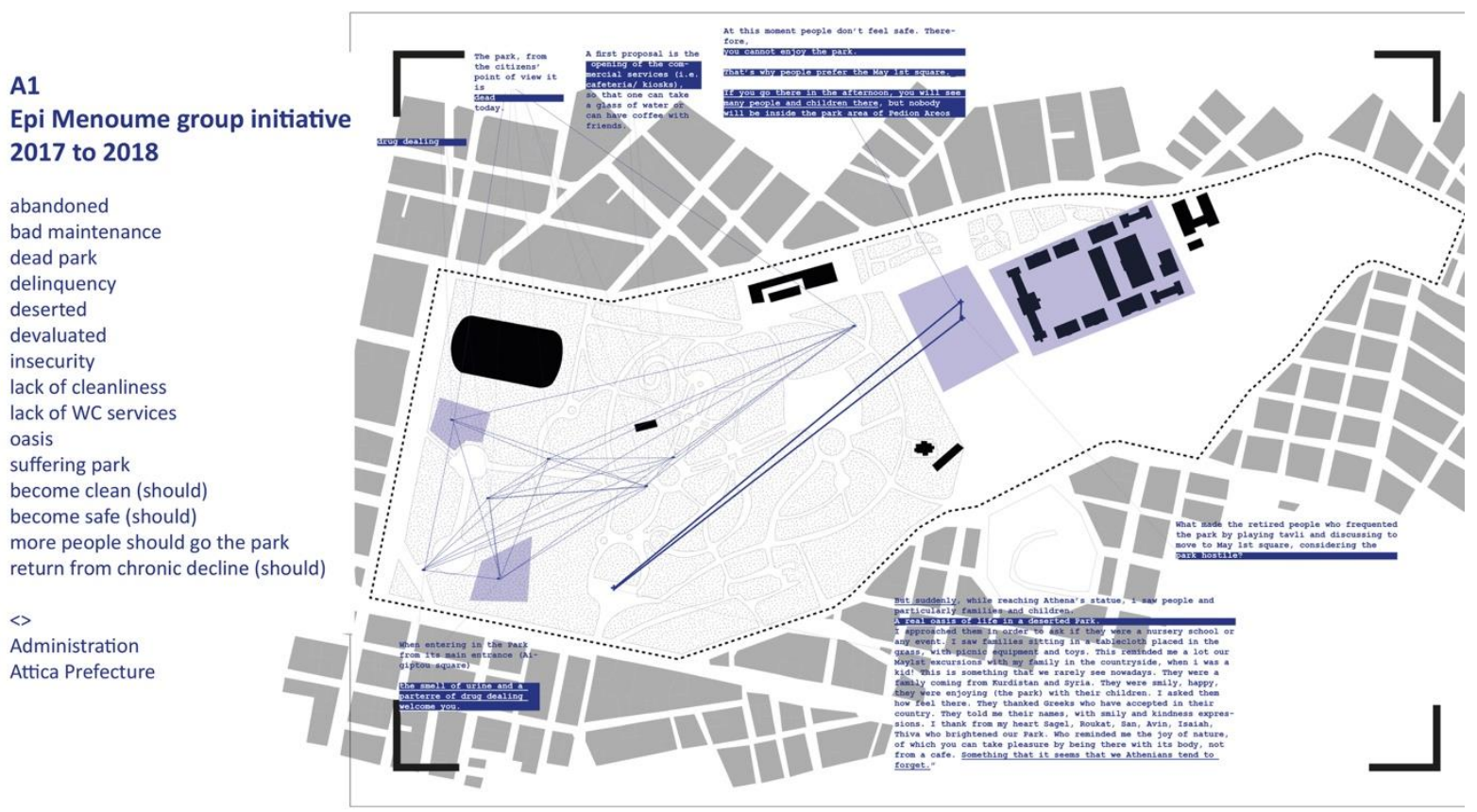

FIGURE 37. Extracted references of the description A1. Please zoom in for (secondary) details on the map. (visualization: Stamatopoulou, 2018)

A13

Tompazis Architectural Office (redevelopment plans report) 2005 to 2008

aged

bad lighting

bad maintenance

benches in bad condition

devaluated

destructions

diversity of trees/ plants insecurity lack of WC services

paths to be organized in hierarchies

vandalized monuments

nice view to Lycabettus Hill

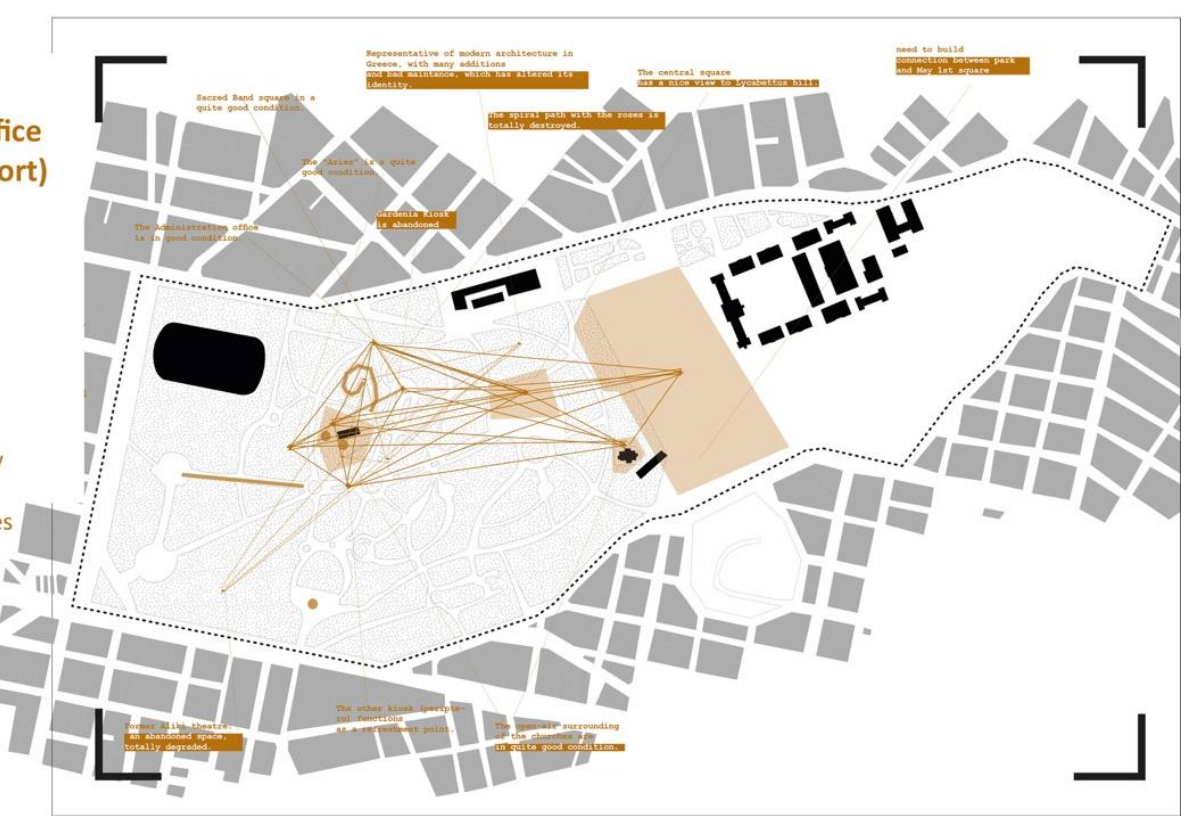

FIGURE 38. Extracted references of the description A13. Please zoom in for (secondary) details on the map. (visualization: Stamatopoulou, 2018) 
A9

Attica Prefecture

2018

abandoned, bad maintenance, in critical condition, become clean, become safe, infrastructure restorations

$<>$

Epi Menoume group initiative

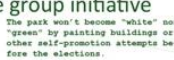



FIGURE 39. Extracted references of the description A9. Please zoom in for (secondary) details on the map. (visualization: Stamatopoulou, 2018)

In Figure 40, the above networks/maps are overlapped, opening the following directions for a strategy composition, integrating multi-perspectivity. Chronological relations give insights into how a field of forces changes with regard to subjects' points of view and agencies over time. And vice-versa, how subjects tend to engage with a park for a defined period of time. This may expand to how the forces change for a type of subject or a relation of subjects in time. Additionally, through the table, it can be monitored-toward an optimized appreciating complexity (Jones 2014)-how a concept changes in time: Regarding problems, their range, repetitions/patterns, and types can be formulated. Observing how problems' diverse manifestations change in time, the design strategy could also expand to prediction and forecasting. It may go deeper on the mechanisms, causes, or the symptoms of conditions generating problems in a systemic way (Rittel and Webber, 1984).

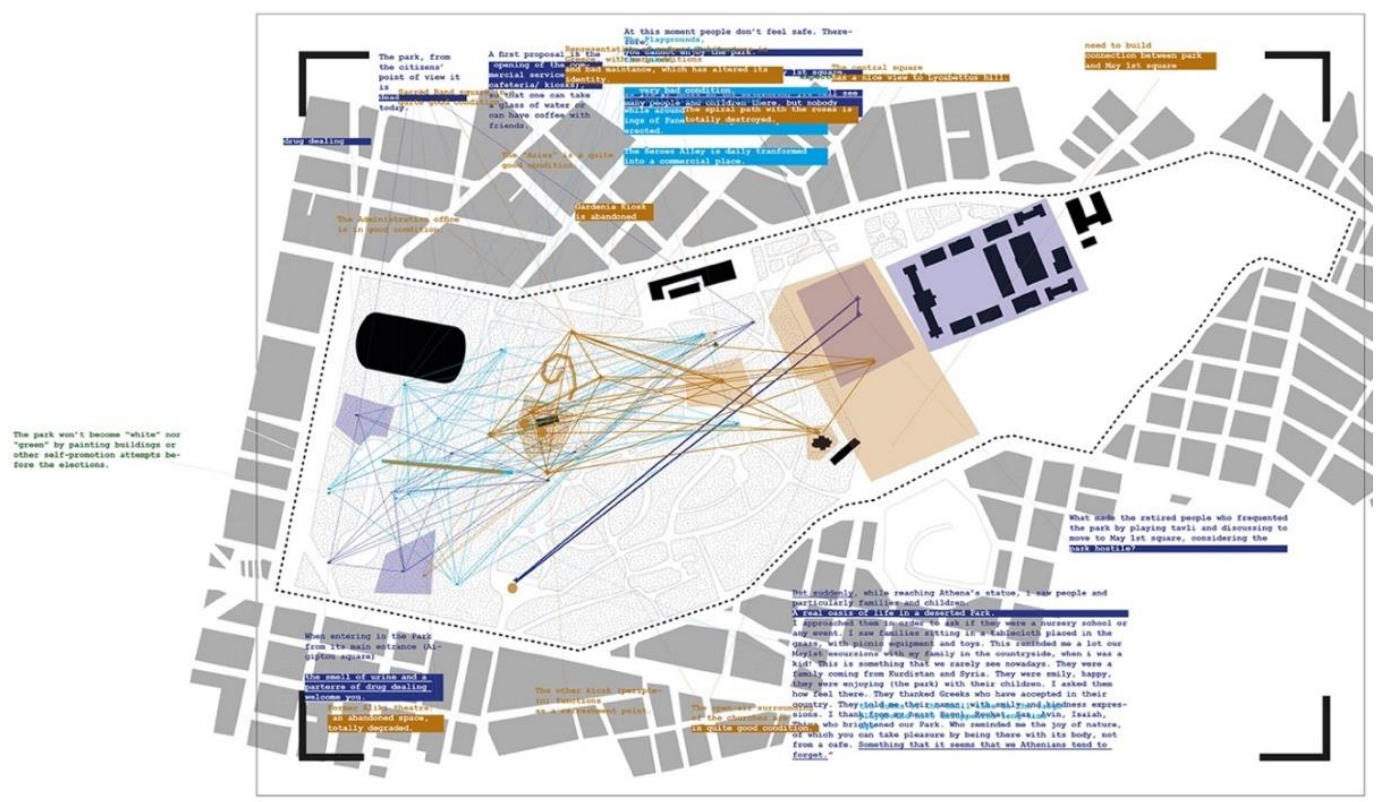

FIGURE 40. Chronological-wise overlapping of mappings A12, A1, A13, and A9 (visualization: Stamatopoulou, 2018). 


\section{Inter-/Intra-parametric design strategies}

Relationally composed objects, like the city, are here argued as assemblages defined by heterogeneous parameters. Diverse subjects, the material territory, and semiotic and framing emergent properties are such parameters in the presented case studies. The in-progress methodology is capable of integrating heterogeneous parameters in analysis and design, while their generative merging is negotiated through diagrammatization operations. By this, the perspective of inter-parametric and intra-parametricoriented design is discussed. In these, parameters are not only co-present; emphasis is placed on how their interactions generate new information. Such interactions enforce intra-parametric design strategies, in which heterogenous parameters merge. Since subjects are parameters, this logic includes inter- or intra-subjective-oriented options for design, depending on subjects' engagement and on the ways their relations are integrated in a process. This perspective is clearer in the Pedion Areos Park case study, in which subjects' diversity enhanced the multi-perspective options of approaching the park. This way, the methodology came in dialogue with the wide field of attempts intended for subjects' engagement, participation, dialogue, and collaboration of more than the designer (i.e. Chora's participatory processes [Bunschoten, 2005]), or co-creation (i.e. Jones, 2018). However, the in-progress methodology is primarily a base of curating non-synchronic interactions among creations, be it triggered or existing, made by multiple subjects. Considering that through the IOM's superimpositions, the boundaries among gathered mappings dissolve yet get distorted, then, through intra-parametric and especially intra-subjective processes, subjects' boundaries are affected as well. This triggers the subjects' and territories' interdependence, and accordingly their fluid borders.

\section{CONCLUSION}

\section{Horizons of potential applications}

The in-progress methodology was designed with the aim of contributing to the field of methods that promote relational thinking and that stress the design of processes. Even if the methodology will be modeled and programmed into a software package or an application in the future, the intended contribution is focused on the methodology of integrating the interconnection of analysis and design through mapping. Therefore, the contribution also depends on the elaboration of the design logic, as with the speculative perspectives explored before. The perspectives' strengths lie in their combinations and synergies, especially in the context of systemic design (Figure 41). At the same time, they are detachable, as they can be integrated into other design processes.

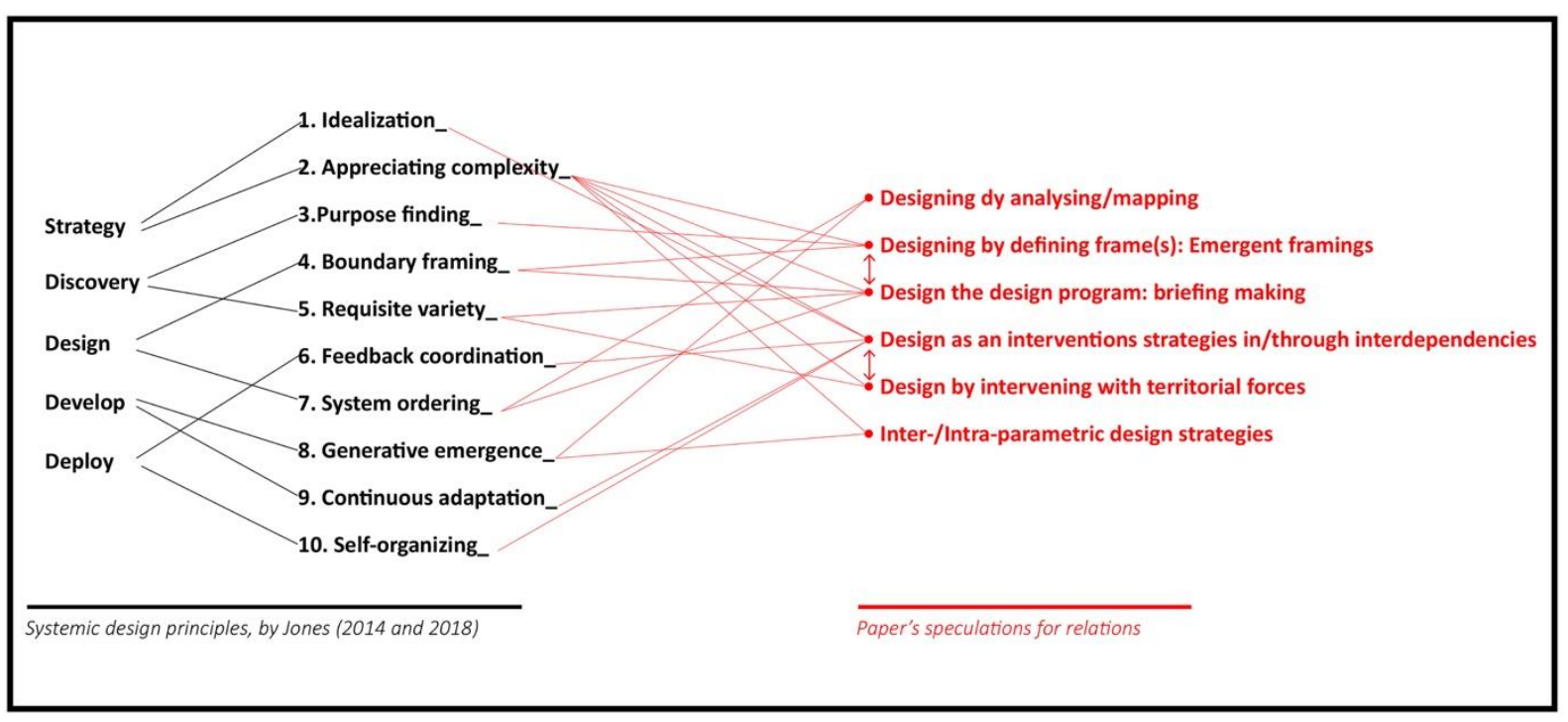

FIGURE 41. The points of logics for the design formulated in the previous section (right side in red) in their (more or less discussed) relations with the 10 principles of systemic design by Jones (2014 and 2018). (visualization: Stamatopoulou, 2020) 
The methodology encourages the holistic approaches of territories, in terms of more-than-the-spatiotemporal dimensions, as this is achieved by the synergy of all the in-between states among semiotic relationality and territoriality. The methodology is also a proposal of logic, while it can function as a tool: By this, its applicability spectrum gets amplified, and in turn the speculative perspective can feed very different contexts and ways of approaching the city, beyond architecture, urban design, and urban planning. The logic of the Interactive Open Map (with optimized visualization) can serve decision-making and policy-making processes. This can be done with different kinds of reasoning. It may operate as a program to work; it can support rational thinking; and it can also unfold as a playful process, like Chora's Scenario Games (Bunschoten, 2007). The latter adds to the perspective of inter-subjective processes, but toward synchronous interactions, which can further evolve into applications for interactive planning. The perspectives discussing strategies can be applied to (urban) redevelopment plans and place branding strategies, for example. Considering the critical aspect of the IOM, these can be counterhegemonic approaches of place-complexity branding. Through its ability to intervene in locations and territorial forces' interdependencies, the methodology can be also used for what is herein called targeted activism, which focuses on changes to networks, instead of random interventions. Such a case would be a network of actions spatio-temporally organized and dispersed in a territory, in a way that, as active forms (Easterling, 2014), become capable of affecting other conditions.

This expanded field of potential applications lies in the variety of the methodology's capacities, but also on its intended flexibility between the so-called bottom-up and top-down logic: The interrelated actions among the different levels, the schemes and data, and the lists and maps traverse these established borders and take advantage of all the in-between opportunities of synergies. In the IOM system, the database and the table give access to the very details of the data, while at the same time the linked map oscillates among top-down versions of diverse territorial scales.

All the perspectives discussed in the paper are at a speculative level and some points remain (too) abstract. Their clarification-maturation, by being attached to the gradual development of the methodology, is expected to evolve through further research, and mainly through more tests. With the experimental practices described in this paper, notice is given to the greater strength of the combinations of conducted tests. Combinations enhance comparisons but also superimpositions; these are generative. For example, the methodology's capacity for inter-scalar analysis is derived from the combinatory approach assumed in this project. Such combinations of tests, having different properties (i.e. territorial-temporal scale, subjects' diversity) as experiments and incorporating criticality, strengthen the methodology's evolution; besides that, they increase the input given by each single experimentation. This criticality is also addressed with regard to the initial form of the tests, since they are put into dialogue. By this, combinatory tests contribute to the feedback-driven process for developing and also training the methodology. Constant critical testing of the methodology is aimed at resisting case-dependence in order to keep its applicability open to different territorial scales, to contexts and intentions, and to time and emergent horizons.

\section{ACKNOWLEDGEMENTS}

An early version of the paper has been presented by the author in the RSD7 Symposium, Relating Systems Thinking and Design 7, hosted at the Department of Architecture and Design (Politecnico di Torino), in Turin, Italy, October 23rd-26th, 2018. A working version of it, under the title Mapping-and-Designing (in) relationally composed fields, has been published, later, in the proceedings of the symposium. Its present evolution into the current paper is a differentiated one. This evolution has been generously supported by the editors of this

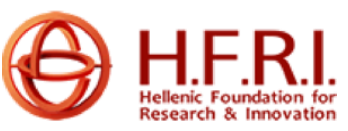

37

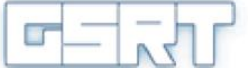

GEMERAI SECRETARIAT FOR

RESEARCH AND TECHNOLOGY 
Special Issue, and particularly the long-term feedback of Dr Marie Davidová, the review process as well as the Systemic Design Association (SDA).

The paper is part of a PhD dissertation in progress, entitled, "The Relationally Composed Object: Description and Design" and is pursued at the School of Architecture, Department III: Architectural Language, Communication and Design, in National Technical University of Athens, Greece. It is supervised by Professor George Parmenides, whose interdisciplinary-oriented expertise on research methodologies has been very catalytic for the development of the presented methodology, beyond the form presented in the paper of this project.

In the period the paper was presented, the PhD research was supported by a scholarship from the General Secretariat for Research and Technology (GSRT) and the Hellenic Foundation for Research and Innovation (HFRI).

\section{Case Studies/Experiments:}

The experiments used and presented have been exclusively elaborated for the purposes of the paper and its broader research context. However, they have been encouraged and partly initiated within other practices of the author.

The first case study as experiment draws data from the collaboration of the author with the postgraduate course (by/of professor George Parmenides) Architecture as a field of research II: On the Conceptual Framework and Body of Research by Design. The course belongs to the division "Research in Architecture: Architectural Design - Space Culture" of the Program Architecture - Spatial Design at the School of Architecture in the National Technical University of Athens (N.T.U.A). The gathered mappings (not shown in any of their original forms in the paper) were made by (in order of appearance in the organized material): Katerina Voulgari, Orfeas Genias, Ismini Epitropou, Ania Zachariadou, Apostolos Zografos, Maria Karaiskou, Stavros Koumoutsos, Irini Koumparouli, Panayotis Koufos, Haris Krekoukiotis, C. Kritikos, Alexandra Mentekidou, Panayota Batalia, Maroula Bacharidou, Stella Boroutzi, Mara Papavasileiou, Maria Papadopoulou, Yota Passia, Panayotis Roupas, Nella Stefanatou, Maria Tassopoulou, Ioanna Fakiri, and Maria Christofi.

This first case study has been widely used as an independent test by the author to present previous phases of the methodology in paper presentations in conferences. Part of it has been used to support a chapter about bottom-up place-branding, included in the following article: Stamatopoulou, A. 2019. Hypertextual places: Mapping relational identities in E. Zantides (Ed.) Semiotics and visual communication III cultures of branding (pp. 320-342). Cambridge Scholars Publishing.

The second case study draws from data by research of the author for the site-specific project by the collective Chto Delat and Anton Kats in Pedion Areos Park. Both the research and the project were supported and organized by Onassis Cultural Centre in Athens in the context of Fast Forward Festival 5. Two of the descriptions included in the data come from interviews made through the collaboration with the cultural anthropologist Pafsanias Karathanasis. 


\section{REFERENCES}

Barad, K. (2007). Meeting the universe halfway: Quantum physics and the entanglement of matter and meaning. University Press.https://doi.org/10.1215/9780822388128

Berkel, van B., \& Bos, C. (Eds.). (1998). Diagram work: Data mechanics for a topological age. (no. 23.). ANY: Architecture New York. https://www.anycorp.com/store/any23

Bunschoten, R. (2003). Stirring still: The city soul and its metaspaces. Perspecta, 34, 56-65. www.jstor.org/stable/1567316

Bunschoten, R. (2005). Points, spirals and prototypes. In P. B. Jones, D. Petrescu, \& J. Till (Eds.), Architecture \& participation (pp. 235-246). Spon Press. https://doi.org/10.4324/9780203022863

Bunschoten, R. (2007). Scenario games. Vital techniques for interactive city planning. In F. Borries, S. Walz, \& M. Böttger (Eds.), Space time play. Computer games, architecture and urbanism: The next level (pp. 384387). Birkhäuser.

Bunschoten, R. (2017). The second skin: From cybernetics to conscious city. In L. Werner (Ed.), Conversations. Cybernetics: State of the art (pp. 92-107). Universitätsverlag der TU Berlin. http://dx.doi.org/10.14279/depositonce-6121

Cilliers, P. (1998). Complexity and postmodernism. Routledge. https://doi.org/10.4324/9780203012253

Cilliers, P. (2001). Boundaries, hierarchies and networks in complex systems. International Journal of Innovation Management, 5(2), 135-47. https://doi.org/10.1142/S1363919601000312

Corner, J. (1999). The agency of mapping. In D. Cosgrove (Ed.), Mappings (pp. 213-252). Reaktion Books.

DeLanda, M. (2016). Assemblage theory. Edinburgh University Press.

Deleuze, G. (1988). Foucault. Translated by H. Seán. University of Minnesota Press.

Deleuze, G., \& Guattari, F. (1987 [1980]). A thousand plateaus. (B. Massumi, Trans.). Bloomsbury.

Easterling, K. (2014). Subtraction. Sternberg Press.

Easterling, K. (2017). Medium design. Strelka Press.

Garcia, M. (Ed.). (2010). The diagrams of architecture. Wiley.

Jones, P. (2014). Systemic design principles for complex social systems. In G. Metcalf (Ed.), Social systems and design (pp. 91-128). Springer. https://doi.org/10.1007/978-4-431-54478-4_4

Jones, P. (2018). Contexts of co-creation: Designing with system stakeholders. In P. Jones \& K. Kijima (Eds.), Systemic design. Theory, methods, and practice (pp. 3-52). Springer. https://doi.org/10.1007/978-4431-55639-8

Massumi, B. (2011). Semblance and event. Activist philosophy and the occurent arts. The MIT Press.

Paez, R. (2019). Operative mapping. Maps as design tools. Actar.

Peirce, C. S. (1931). Collected papers of Charles Sanders Peirce. (6 volumes). C. Hartstone \& P. Weiss (Eds.). The Belknap Press.

Rittel, H. W. J., \& Weber, M. (1973). Dilemmas in a general theory of planning. Policy Sciences, 4, 155-169. https://doi.org/10.1007/BF01405730

Sevaldson, B. (2009). About systems oriented design. Systems Oriented Design. https://www.systemsorienteddesign.net/index.php/sod/about-sod

Sevaldson, B. (2010). Discussions \& movements in design research. FormAkademisk-Research Journal of Design and Design Education, 3(1). https://doi.org/10.7577/formakademisk.137

Sevaldson, B. (2011). GIGA-mapping: Visualisation for complexity and systems thinking in design. Nordic Design Research Conferences, Making Design Matter. Helsinki, Finland: NORDES. https://pdfs.semanticscholar.org/872a/0d2f08db4442e78fd775acfbfa008cb16eb6.pdf 
Sevaldson, B. (2017). ZIP - Analysis. Systems Oriented Design. https://www.systemsorienteddesign.net/index.php/zip

Sevaldson, B. (2018a). Visualising complex design: The evolution of Gigamaps. In P. Jones \& K. Kijima (Eds), Systemic design. Theory, methods, and practice (pp. 243-269). Springer. https://doi.org/10.1007/978-4431-55639-8

Sevaldson, B. (2018b). Library of systemic relationships. Systems Oriented Design. https://www.systemsorienteddesign.net/index.php/tools/types-of-systemic-relationships

Schön, D. (1983). The reflective practitioner: How professionals think in action. Temple Smith.

SI, (1981), Definitions. In K. Knabb (Ed.) Situationist International Anthology. (Revised and Expanded Edition, pp. 51-52). Bureau of Public Secrets. (Original work published 1958). https://libcom.org/files/Situationist\%20International\%20Anthology.pdf

Zdebik, J. (2012). Deleuze and the diagram. Aesthetic threads in visual organization. Bloomsbury.

\footnotetext{
${ }^{1}$ The term relations is used in the paper instead of this of relationships. The former is considered to particulary refer to potential associations among very diverse things, but most important selection criterion is that it matches to how the cited philosophy conceptualizes it.

${ }^{2}$ The term locations is of frequent use in the paper. When not specified otherwise, it is an open/abstract reference to potentially diverse types of physical territories such as areas, sites and streets, or even networks.

${ }^{3}$ Both Paez (2019) and Corner (1999) are aligned with Deleuze's and Guattari's distinction between a static tracing and the more performative mapping (Deleuze and Guattari 1987). In this distinction, tracing and mapping are not binaries, they are supplementary as put by Deleuze and Guattari (1987:3): “... the tracing should always be put back on the map." This distinction matches to this with consideration for the representational map, that is the tracing, and the non-representational map, that is Deleuze's and Guattari's map, which is synonymous to Deleuze's (1986) diagram. In this paper, the first distinction was taken into account, but it does not adopt the terms "tracing" and "mapping." Mapping is used in both ways, while its diagrammatic aspects stress its operative and non-representational capacities, as further explained in the paper.

${ }^{4}$ Emergence of new is also discussed in systemic design principles through Generative Emergence (Jones, 2014: 117).

${ }^{5}$ Massumi (2011: 14) remarks that "diagramming is the procedure of abstraction when it is not concerned with reducing the world to an aggregate of objects, but quite the opposite, when it is attending to their genesis." In this argument he refers to relevant points about the diagram and diagramming from Peirce and broadly from Deleuze.

${ }^{6}$ This entity is interpreted in the paper as familiar with DeLanda's (2016:10) emergent whole: He distinguishes what he names "emergent wholes" from "wholes" with regard to the role of the parts of the whole. Parts of wholes adopt features of the whole. Parts of emergent wholes (which result from relationships of exteriority) are autonomous and detachable.

${ }^{7}$ The diagram has been widely adopted and interpreted by several architects and theorists, as extensively analyzed by Marc Garcia (2009) and in the $23^{\text {rd }}$ volume of ANY magazine, edited by Ben van Berkel and Caroline Bos (1998). Reviewing the paper's relationships with them-both theoretically as well as with regard to how diagram is applied-is out of its frame. The paper is targeted to the presentation of a broader methodology, while directly drawing from the original key resources about the diagram, namely Peirce, along with Deleuze and Guattari.

${ }^{8}$ As before, a literature review for this is out of this paper's context, since it requires a paper dedicated to such an objective.

${ }^{9}$ The design of the park is composed of three disjoined layers-systems: the system of surfaces, the system of points (folies) and the system of lines (cinematic promenade). The three systems are not superimposed in the masterplan; they set potentialities of a superimposition unfolding through on-site, real-time, life relations.

${ }^{10}$ In Urban Gallery methodology, a targeted territory is partitioned in smaller areas. Different groups of individuals undertake the formation of the database of each area through mini scenarios. By this, the database is composed of different points of view. The total of the collected information covers the larger territory (the partitioned one) than that of each approach's individual focus. Therefore, information is elicited by zooming in, and the collected totality offers a zoom-out view, which is inter-subjectively formed. This is a feature that the in-progress methodology has as well.

${ }^{11}$ In Urban Gallery, "intertwining" and "connectivity" concern operations among layers of prototypes (Bunschoten, 2003).

12 This is also clarified by Bunschoten (2005: 246) as an "endless proliferation in a larger area."

${ }^{13}$ Peter Jones (drawing from Bousbaci, 2008; cited in Jones, 2014) classifies the plurality of design methods into four generations and paradigmatic shifts (Jones, 2014: 98-99). According to this view, they begin from the more rational, engineering-oriented approaches of the 1960s, including Simon and Fuller; in the 1970s they turn to pragmatics through the so-called wicked problems as well as Rittel and Chris Jones; in the 1980s phenomenological accounts giving priority to the social appeared, along with participatory and user-centered rhetoric of design; the 2000s are linked to "generative, empathetic and transdisciplinary" methods.

${ }^{14}$ Peter Jones (2014) has gathered and elaborated the principles through a process model that interrelates exploration, formation, and evaluation composed of five phases: strategy, discover, design, develop, deploy (Jones, 2014: 107-108).

${ }^{15}$ This principle also expands also to Reframing options.

${ }^{16}$ Gigamaps are developed as visual thinking tools and have the form of large-scale, extensive mappings. They represent and organize dense information, while they manifest in a wide variation and in different types (Sevaldson, 2011). Accordingly, they have a large variety of features that expand to a spectrum of applications. Among the features, the potential for multiple connections, meanings, interpretations, and layerings across diverse scales, are here remarked. This includes the challenge of how the numerous diverse relationships (i.e. structural relationships, emotional relationships, negative relationships, positive relationships, spatial proximity, and temporal proximity) among things can be noted
} as information not separately accessed (Sevaldson, 2018b). 
17 Analysis is extensive and made by the author without the use of any software for data analysis.

${ }^{18}$ In the general level of the development of methodology (general rules), and considering its targeting as a research analysis and design tool, the organization and the classification of properties is not predefined. Namely, each test is flexible enough to decide its parameters without constraints. There are two reasons for this openness. First, parametrization, based on fixed classification, might regulate potentiality and predefine the outcome. Second, a parametrization has agency. It is attached to the context of the methodology application (i.e. design intentions).

${ }^{19}$ No software is used. The codification, the noting of the data, and the formation of the maps and the tables is also done manually. Reflection has benefited from this process.

${ }^{20}$ Based on Peirce's (1931) triadic model, such concepts correspond to the first term, namely the representamen. This is the filter through which the subjects attribute meanings, and broadly significations to the city it maps.

${ }^{21}$ The latter triggers thoughts for non-human-centered properties, and how the methodology could further expand the intended multicentrality by integrating in the first-level data related to non-human actors.

22 Peirce's (1931) triangle is a way to organize them, considering the concepts of the commons and of the Otherness as kinds of representamens, and all the meanings attributed through them as interpretants.

${ }^{23}$ Here, "problems" corresponds to Peirce's (1931) representamen and the "significations" referred to Peirce's interpretants. 